\title{
Una nueva estimación del índice del costo de vida, Argentina 1912-1932
}

\author{
Cecilia T. Lanata Briones ${ }^{1}$ \\ Teaching Fellow, University of Warwick e Investigadora Adjunta del Centro Interdisciplinario para \\ el Estudio de Políticas Públicas. \\ clanata@gmail.com
}

Artículo recibido: 02 de octubre de 2018. Aprobación final: 22 de febrero de 2019.

\section{Resumen}

$\mathrm{Al}$ ser concebidas como reflejos o aproximaciones a la realidad, las estadísticas ayudan a comprender hechos porque objetivan fenómenos. Esta idea se basa en la premisa de que las herramientas estadísticas son hechos incontestables y apolíticos. Sin embargo, la cuantificación y sus resultados no son objetivos. Para determinar el fenómeno a medir y el objetivo de la cuantificación primero se necesitan definiciones. Por lo tanto, las estadísticas están sujetas a debates en torno a sus métodos, interpretación y uso. Utilizando la primera estimación del índice de costo de vida (ICV) argentino y siguiendo la metodología de de-construcción/ construcción/re-construcción de estadísticas, este artículo estudia cómo se generan las mismas. En la fase de de-construcción, el trabajo analiza varios informes para determinar cómo se estimó dicho ICV, elaborado por Alejandro Bunge. La etapa de construcción analiza la metodología del índice y determina los problemas del mismo, que son consecuencia de las suposiciones y los métodos utilizados, en base a los datos disponibles en ese entonces. Por último, el ICV se re-construye corrigiendo sus principales problemas, utilizando la información disponible para Bunge, con el fin de demostrar cómo diferentes supuestos resultan en diferentes series. Por ello, se genera una nueva estimación del ICV para el período 1912-1932. 


\title{
A new estimate of the Argentine Cost of Living Index, 1912-1932
}

\begin{abstract}
Statistics contribute to the understanding of events by objectifying phenomena, as they are perceived to reflect or be an approximation of reality. This perception is based on the premise that statistical tools are straightforward, apolitical facts. However, quantification and its results are not objective. Definitions are needed beforehand to determine the phenomenon to be measured and the aim of the quantification. Thus, statistics face debates on methods, interpretation and use. Using the first estimate of the Argentine cost of living index (COLI) as a case study and following the process of de-construction/construction/re-construction of statistics, this article studies how statistics are made. In the de-construction phase, the paper analyses various reports to arrive at an understanding of how the COLI, elaborated by Alejandro Bunge, was originally estimated. The construction stage discusses the methodology that he used. The pitfalls that come from the assumptions and methods underlying the index are demonstrated using data available at that time. Lastly, the COLI is re-constructed by correcting its main pitfalls using the information available when the series were initially developed to depict how different assumptions result in different series. A new estimate of the COLI is elaborated for the period 1912-1932.
\end{abstract}

Keywords: Price index, History of Statistics, Alejandro E. Bunge, Argentina.

Las estadísticas son una forma de conocimiento y una fuente de poder (Senra, 2011). Deben reflejar la realidad o ser una aproximación de la misma. Sin embargo, "la "realidad" es informada por un entramado bastante inconsciente de varias actitudes hacia la realidad” (Desrosières, 2001:339). Las estadísticas reflejan la realidad y a la vez la construyen: "son objetivaciones complejas que revelan realidades idealizadas previamente pensadas” (Senra, 2011: 52). Para generarlas, se necesitan definiciones que determinen el fenómeno a medir y el objetivo de la cuantificación, decisiones que son tomadas por (grupos de) individuos que actúan según ideas y objetivos propios. Todas las estadísticas se encuentran sujetas a una discusión sobre métodos, interpretación y uso. Aunque pueden percibirse como hechos incontestables, la cuantificación y sus resultados no son objetivos. Las agencias estadísticas son centros de investigación que se adecuan a reglas científicas. Como productoras de estadísticas públicas, están sujetas a reglas, valores y restricciones de la esfera pública (Schwartzman, 1997: 9). Las estadísticas no sólo crean y desarrollan conocimiento, también contribuyen al diseño de políticas y se utilizan en estudios académicos. Para D.C.M. Platt, las fuentes de información de las estadísticas históricas se remontan a un pequeño número de autores y estimaciones fundacionales, que son débiles y a veces carecen de referencias bibliográficas. Según él, estadísticas de dudosa calidad y veracidad viajan en el tiempo para convertirse en saber convencional. A pesar de cuestionar todo tipo de estadísticas, Platt sostiene que las estadísticas públicas -generalmente consideradas superiores porque "las cifras oficiales apelan a las mentes oficiales” (1989: 7)- reflejan la política del gobierno. 
Por ello, el análisis de la producción de estadísticas públicas es un estudio en sí mismo. Este trabajo examina la generación de estadísticas utilizando la primera estimación del índice del costo de vida (en adelante, ICV) argentino -publicada por Alejandro E. Bunge en la Revista de Economía Argentina (en adelante, REA) en 1918 y por la Dirección General de Estadística de la Nación (en adelante, DGEN) en 1924- siguiendo la metodología de de-construcción/construcción/ re-construcción de estadísticas (Lanata Briones, 2016). Este procedimiento en tres pasos -que aplicado al ICV argentino incluye un análisis detallado de la forma en que se produjo dicha estimación y genera un nuevo índice- brinda una nueva dimensión a la literatura que estudia la historia de las estadísticas públicas en general y a la nacional en particular, al responder las siguientes preguntas: ¿cómo se elaboró el ICV de Bunge?, ¿cuáles son las implicancias de las suposiciones usadas en la estimación?, ¿cuáles son sus puntos fuertes y sus problemas?, y ¿cómo se comparan las series re-construidas con la oficial? La metodología utilizada surge de la necesidad, planteada por Platt, de cuestionar las estadísticas históricas ya que son "fuentes históricas" (Tooze, 2008: 683), y combina aspectos de la sociología de la cuantificación con la sociología del conocimiento económico. La socio-historia de la cuantificación analiza cómo se desarrollan las estadísticas conjuntamente con los esfuerzos públicos y privados para organizar y controlar la sociedad (Desrosières, 1993; Hacking, 1990; Porter, 1995), focalizándose en las razones políticas detrás su generación (Alonso y Starr, 1987; Anderson, 1988; Curtis, 2001; Loveman, 2014; Tooze, 2001, entre otros). Esta disciplina es una "búsqueda teórica y práctica" (Prévost y Beaud, 2012:6), en la cual aspectos científicos, administrativos y políticos están estrechamente relacionados. Las estadísticas "proporcionan una descripción resumida de [situaciones] para que puedan recordarse y usarse como base para la acción" (Desrosières, 1993: 13). Para tal fin, se debe construir, junto con el procesamiento matemático, un espacio político de equivalencia y codificación. Alain Desrosières concibe a las estadísticas como conceptos formales y sintéticos que son "el resultado de una gestación histórica marcada por vacilaciones, rectificaciones e interpretaciones conflictivas" (1993: 2). Ted Porter (1995) sostiene que los números son una estrategia de comunicación que se presenta como un discurso altamente disciplinado y desvinculado del conocimiento de las personas que los producen. Es decir, las estadísticas son el resultado de construcciones y convenciones, sujetas a normas establecidas por individuos.

Los ICV son instrumentos de conocimiento económico. La sociología del conocimiento económico estudia las vinculaciones entre ideas, individuos e instituciones (Furner y Supple, 1990), destacando la relevancia del contexto, el cual influye sobre qué preguntas cobran relevancia y cómo éstas se responden, a la vez que determina cuáles son los instrumentos de conocimiento que se producen (Fourcade, 2009). Los problemas sociales y económicos impactan en la generación de conocimiento económico (Hayes, 2011). Dicho conocimiento y su transformación en políticas e instrumentos de política están relacionados con las tradiciones intelectuales nacionales, a pesar de la necesidad de sistematización (Fourcade, 2009; Neiburg, 2006). La construcción de estadísticas es un proceso social que sigue y aplica reglas. Estas acciones, al ser decisiones tomadas por 
individuos, implican valoraciones. Los individuos están inmersos en una sociedad: las fuerzas políticas y económicas influyen en las identidades, actividades y proyectos profesionales (Fourcade, 2009).

A pesar estudios recientes (Cuesta, 2016b; Daniel y Heredia, 2014; Daniel y Lanata Briones, 2019; Jany-Catrice, 2018; Lanata Briones, 2016, en prensa a, en prensa b; O'Neill, Ralph y Smith, 2017; Searle, 2015; Stapleford, 2009; Touchelay 2015; Tooze, 2001), las estadísticas económicas y socio-laborales están menos estudiadas que las demográficas (Tooze, 2001: 3). Este trabajo analiza la generación de ICV, ${ }^{2}$ un indicador que permite la indexación, que es "el uso más extremo de las estadísticas económicas en la vida política” (Stapleford, 2009: 5). La indexación hace que las tareas del gobierno sean técnicas, administrativas e impersonales, eliminando la responsabilidad política en cualquier actividad relacionada con ese procedimiento. Por lo tanto, los ICV tienen una doble naturaleza: describen eventos empíricos pasados y prescriben el comportamiento futuro (Neiburg, 2006: 614). Los ICV no sólo corroboran la existencia de aumentos de precios. Según Hayes (2011: 107), también transforman sustancialmente la naturaleza del fenómeno inflacionario y el contexto en el cual se toman decisiones. Con el florecimiento de las estadísticas económicas entre 1870 y la década de 1950, los ICVs se convirtieron en aspectos fundamentales de una economía nacional (Tooze, 2001:4-11). La Primera Guerra Mundial fue un momento crucial en la historia de los ICV, ya que los cambios en el contexto económico debían comprenderse, contabilizarse y compararse. Desde la Segunda Guerra Mundial, los índices de precios tienen funciones económicas, disociadas de sus funciones sociales previas, lo cual influye en la relación entre clases sociales y estado, así como en su construcción (Hayes, 2011).

Los informes estadísticos determinan "la legitimidad de ciertas visiones del orden social" (Scott, 1988: 115). Si se usan sin cuestionar, persiste una idea específica de la economía y de la ciencia estadística como fundamentalmente objetiva, y sus usuarios "se convierten involuntariamente en partícipes de la política de otra época” (Scott, 1988: 137). Como generadores de conocimiento económico, estos documentos deben ser examinados con referencia al contexto para no ser malinterpretados (Harper, 1998), convirtiéndose en objetos de estudio. Junto con las estadísticas, son el objeto de estudio de este trabajo y las principales fuentes primarias utilizadas. Los informes y artículos estadísticos oficiales "periféricos" donde también se explica el índice, los escritos de Bunge, otras publicaciones del aparato estadístico nacional, folletos, libros y artículos de revistas académicas de la época también constituyen el cúmulo de fuentes consultadas. ¿Qué implica esto? Hasta el momento, el ICV argentino ha sido tratado como una ciencia ya constituida (ready-made science) (Latour, 1987). Este trabajo, por el contrario, lo considera como una ciencia en construcción (science in the making), con el fin de obtener una imagen completa y coherente del indicador y del proceso detrás de su elaboración.

$2 \mathrm{Al}$ analizar el indicador que midió los movimientos de precios en la primera mitad del siglo XX, es históricamente apropiado usar el término ICV porque sus desarrolladores y usuarios se refirieron a dicha estadística con ese nombre. A pesar de que difiere de su significado actual, ICV será el término utilizado en este trabajo. 
Después de esta introducción, en la segunda sección se explican la metodología utilizada y el por qué del caso de estudio. Siguiendo tal metodología, la tercera parte de este trabajo de-construye el ICV de Bunge mientras que la cuarta lo construye y la quinta lo re-construye. La última parte contiene algunas reflexiones finales.

\section{La metodología de de-construcción/construcción/re-construcción y el caso del ICV argentino}

Al combinar las premisas de la sociología de la cuantificación y de la sociología del conocimiento económico con la necesidad de cuestionar los números públicos, este trabajo analiza el primer ICV argentino utilizando la metodología de de-construcción/construcción/re-construcción de estadísticas (Lanata Briones, 2016). Esta dinámica y flexible metodología en tres etapas puede aplicarse a cualquier estimación estadística. El énfasis puesto en cada fase del proceso y la extensión del análisis de cada etapa dependen de la estimación. En el caso del primer ICV argentino, su metodología es difícil de entender leyendo únicamente el informe en el que se publicó originalmente (Bunge, 1918). Por ello, deben analizarse artículos periféricos (Bunge, 1919; DGEN, 1924; Valle y Ferrari, 1920). La fase de de-construcción sintetiza la información provista por los artículos centrales y periféricos para establecer la metodología y las características del ICV. Dicha etapa es seguida por un análisis del contexto, los usos, la recepción y el significado de la estimación; y por un estudio de los problemas del índice. Esta es la fase de construcción, que se lleva a cabo para comprender por qué y cómo cada estimación fue producida y utilizada. Este análisis no constituye un juicio de valor de los métodos y suposiciones en base a criterios actuales, ya que las metodologías se han vuelto cada vez más sofisticadas. Es un estudio de los supuestos y de los métodos, utilizando los datos disponibles para aquellos que produjeron las estimaciones. La segunda etapa es una investigación de las personas e instituciones involucradas y la metodología detrás de la producción de los índices en su contexto económico y social. La fase de re-construcción tiene dos partes. Por un lado, el ICV se re-construye corrigiendo sus principales problemas, utilizando la información disponible en el momento en el cual la serie fue desarrollada con el fin de re-construirla lo más fielmente posible, y se confronta con la estimación oficial, demostrando cómo diversas suposiciones generan series diferentes. Por otro lado, se comparan las características del ICV argentino con las de otros índices que fueron publicados en la misma época. Este artículo se concentra de forma muy sintética en la primera fase, en los problemas del ICV de Bunge (etapa de construcción) y en la primera instancia de la etapa de re-construcción. ${ }^{3}$

Este trabajo investiga los orígenes del ICV argentino durante la primera mitad del siglo XX, enriqueciendo la historia de los ICV y de los sistemas estadísticos nacionales (Anderson, 1988; Camargo, 2007; Curtis, 2001; Loveman, 2014;

3 Las ideas, instituciones y el individuo detrás de esta primera estimación se analizan en Lanata Briones (en prensa a). Lanata Briones (2016, en prensa a) compara aspectos del ICV de Bunge con los índices de Gran Bretaña, Alemania y Estados Unidos. 
Patriarca, 1996; Tooze, 2001, entre otros). Específicamente, el artículo es una contribución a la literatura que estudia al aparato estadístico argentino. Desde la sociología de la cuantificación, Claudia Daniel (2009), Hernán González Bollo (2014) y Hernán Otero (2006) analizan la formación, consolidación y trayectoria de las estadísticas públicas argentinas y cómo las mismas dieron forma a la política gubernamental. Con la utilización de la metodología de de-construcción/ construcción/re-construcción de estadísticas, esta investigación extiende los aportes de estos autores dada la centralidad del índice dentro del aparato estadístico y la importancia de Alejandro E. Bunge en el mismo (González Bollo, 2012 y 2014; Pantaleón, 2009: 25). Los autores de la sociología de la cuantificación interpretan el contexto y las cifras centrándose principalmente en la política de las estadísticas y analizan los informes estadísticos, pero no profundizan en la construcción metodológica de los números. Examinar las agencias estadísticas como un sistema de poder y conocimiento debe complementarse con el estudio de las estadísticas como fuentes históricas, como construcciones y usos de hechos particulares, analizando con detalle las cifras producidas por el sistema estadístico nacional.

Para Stapleford (2009), no existe una metodología universalmente válida y correcta para estimar los ICV porque, al construirlos, existen ambigüedades, y los métodos utilizados dependen de los usos esperados del índice. Además, determinar una metodología para estimar estos índices genera preguntas y críticas para las cuales no existen respuestas apolíticas. Por lo tanto, los argumentos que surgen desde la sociología de la cuantificación y desde la sociología del conocimiento económico validan la elección del ICV argentino.

Especificidades del caso de estudio también explican la elección del ICV argentino. El indicador fue publicado por primera vez en sintonía con otros, pero -a diferencia de sus contrapartes estadounidense, británica y alemana- entre 1918 y 1935 fluctuó entre ser una estadística privada y una pública (Lanata Briones, 2016). Su primera difusión fue en 1918 en la REA, y en 1924 la DGEN lo publicó oficialmente. Sus actualizaciones posteriores formaron parte, una vez más, de la REA. Un decreto presidencial de 1932 exigía la elaboración de una nueva estimación, y entre 1935 y 2007 el ICV fue una estadística pública. ${ }^{4}$ Asimismo, la inflación tiñe la historia económica argentina, especialmente desde la segunda mitad del siglo XIX, y particularmente con los episodios hiperinflacionarios de 1989 y $1991 .{ }^{5}$ De hecho, el índice se ha vuelto tan relevante que se ha convertido en un indicador que da cuenta del éxito o fracaso de la gestión económica del gobierno de turno. Los argentinos han aprendido a convivir y a lidiar con los aumentos de precios. A pesar de estas características, y a diferencia de lo que sucede con el producto interno bruto -para el cual existen estimaciones (además de las oficiales) para diferentes períodos ${ }^{6}-$ y con las cifras del comercio exterior (Kuntz-Ficker y Rayes,

4 Daniel y Lanata Briones (2019) explican lo sucedido con el índice entre 2007 y 2015.

5 Damill y Frenkel (1990) y Vitelli (1986), entre otros, examinan la "historia reciente" de la inflación. Para un análisis del siglo XIX ver, por ejemplo, Amaral (1995) e Irigoin (2000).

6 Una de las primeras personas en cuestionar las estimaciones del PIB argentino fue Randall (1976). Un resumen de las series existentes se encuentra en Francis (2013) y Harriague y Rayes (2018). 
2017; Tena-Junguito y Willebald, 2013), la historiografía de la primera mitad del siglo XX ha utilizado sistemáticamente el ICV oficial sin cuestionarlo, como se muestra en el cuadro $1 .^{7}$

Cuadro 1. Trabajos seleccionados sobre la Argentina según la fuente del ICV que utilizan

\begin{tabular}{|c|c|c|c|c|c|c|c|c|c|c|c|c|c|}
\hline \multirow[b]{3}{*}{ Trabajos } & \multicolumn{13}{|c|}{ Fuentes del ICV utilizadas } \\
\hline & \multicolumn{4}{|c|}{ Primaria (P) } & \multicolumn{9}{|c|}{ Secundaria(S) } \\
\hline & \begin{tabular}{|l|} 
REA/ \\
BUNGE
\end{tabular} & $\begin{array}{l}\text { DNT } \\
/ 1 / 5\end{array}$ & DNEC & Villanueva & \begin{tabular}{|l|} 
Anuario \\
Geogr ófico \\
Argentino \\
\end{tabular} & \begin{tabular}{|l|} 
della \\
Paolera y \\
Ortiz \\
\end{tabular} & Dorfman & \begin{tabular}{|l|} 
Díaz \\
Alejandro \\
(1981)
\end{tabular} & $\begin{array}{l}\text { Gerchunoff } \\
\text { y Llach }\end{array} \mid$, & IEERAL & $\left|\begin{array}{l}\text { Véganzonès } \\
\text { y Winograd }\end{array}\right|$ & Williamson & $\left\{\begin{array}{l}\text { Williamson } \\
\text { y Taylor }\end{array}\right.$ \\
\hline Cortés Conde, R. (1979). El progreso & $\mathbf{P}$ & $\mathbf{P}$ & & & & & & & & & & & \\
\hline Di Tella, G. y Zymelman, M. (1967). Las etapas & $\mathbf{P}$ & $P$ & & & & & & & & & & & \\
\hline \begin{tabular}{|l|} 
Diaz Alejandro, C. (1970) 1983. Essays \\
\end{tabular} & & & $\mathbf{P}$ & & & & & & & & & & \\
\hline Gaudio, R.y Pilone, J. (1983). El desarrollo & & $\mathbf{P}$ & & & & & & & & & & & \\
\hline Germani, G. (1955) 1980. Estructura & & $P$ & & & & & & & & & & & \\
\hline Harriague, M. M. y Rayes, A. (2018). Fuentes & $\mathbf{P}$ & $P$ & $\mathbf{P}$ & $\mathbf{p}$ & & & & & & & & & \\
\hline Horowitz, J. (1990). Argentine Unions & & $P$ & & & & & & & & & & & \\
\hline Iñigo Carrera, J. (2007). La formación & $\mathbf{P}$ & $\mathbf{P}$ & & & & & & & & & & & \\
\hline Matsushita, H. (1983). Movimiento obrero & & $\mathbf{P}$ & & & & & & & & & & & \\
\hline Murmis, M. y Portantiero, J.C. (1971). Estudios & & $P$ & & & & & & & & & & & \\
\hline Vázquez Presedo, V. (1988). Estadisticas & & & $\mathbf{P}$ & & & & & & & & & & \\
\hline Anuario Geogrä́fico Árgentino $\left(\overline{194} \overline{1} \overline{1}^{-}\right.$ & & $\overline{\mathrm{p}}$ & & & & & & & & & & & \\
\hline Díaz Alejandro, C. (1981). Tipo de cambio & & & $\mathbf{P}$ & & & & & & & & & & \\
\hline Dorfman, A. (1970). Historia de la industria & & $P$ & & & & & & & & & & & \\
\hline Villanueva, J. (1964). The Inflationary Process & & & $\mathbf{P}$ & $\mathbf{P}$ & & & & & & & & & \\
\hline \begin{tabular}{|l|l} 
Williamson, J. (1995). The Evolution \\
\end{tabular} & $P$ & $P$ & & & & & & & & & & & \\
\hline della Paolera, G. y Ortiz, J. (1995). Dinero & $\mathbf{P}$ & & & & & & & $\mathbf{s}$ & & & & & \\
\hline IEERAL (1986). Estadisticas & & & & & & & & $\mathbf{s}$ & & & & & \\
\hline Gerchunoff, P. y Llach, L. (1998). El ciclo & & & & & & $\mathrm{s}$ & & & & & & & \\
\hline $\begin{array}{l}\text { Véganzonès, M. y Winograd, C. (1997). } \\
\text { Argentina in the 2oth Century }\end{array}$ & & & & & & & & & & $\mathbf{s}$ & & & \\
\hline Williamson, J. y Taylor, A. (1994). Convergence & & & & & & & & & & & & $\mathbf{s}$ & \\
\hline Cortés Conde, R. (2009). The Political Economy & & & & & s & & & & & & & & \\
\hline \begin{tabular}{|l|} 
Ferreres, 0. (2005). Dos Siglos \\
\end{tabular} & & & & & $\mathbf{s}$ & & & & & & & & \\
\hline $\begin{array}{l}\text { Vence Conti, A. y Cuesta, E. M. (2014). } \\
\text { Politicas laborales y salarios }\end{array}$ & $\mathbf{P}$ & $\mathbf{P}$ & $\mathbf{p}$ & & $\mathbf{s}$ & & & & & & & & \\
\hline $\begin{array}{l}\text { della Paolera, G., Irigoin, A. and Bozzoli, G. } \\
\text { (2003). Passing the buck }\end{array}$ & & & & & & $\mathbf{s}$ & & & & & & & \\
\hline $\begin{array}{l}\text { della Paolera, G. and Taylor, A. (2001). } \\
\text { Straining at the Anchor }\end{array}$ & & & & & & $\mathbf{s}$ & & & & & & & \\
\hline Shipley, R. (1977). On the outside & $\mathbf{P}$ & & & & & & $\mathbf{s}$ & & & & & & \\
\hline Cortés Conde, R. (1994). Econom ia Argentina & & & & & & & & $\mathbf{s}$ & & & & & \\
\hline $\begin{array}{l}\text { Sturzenegger, A. y Moya, R. (2003). Economic } \\
\text { cycles }\end{array}$ & & & & & & & & & $\mathbf{s}$ & & & & \\
\hline $\begin{array}{l}\text { della Paolera, G., Taylor, A.y Bozzoli, G. } \\
\text { (2003). Historical statistics }\end{array}$ & & & & & & & & & & $\mathbf{s}$ & & & \\
\hline Vitelli, G. (1999). Los dos siglos & & & & & & & & & & $\mathbf{s}$ & & & \\
\hline $\begin{array}{l}\begin{array}{l}\text { Galiani, S. y Gerchunoff, P. (2003). The labour } \\
\text { market }\end{array} \\
\end{array}$ & & & & & & & & & & & $\mathbf{s}$ & & \\
\hline $\begin{array}{l}\text { Gerchunoff, P. y Aguirre, H. (2006). La } \\
\text { economía }\end{array}$ & & $\mathbf{P}$ & & & & & & & & & & $\mathbf{s}$ & \\
\hline Salvatore, R. (2010). Better-off & & & & & & & & & & & & & $\mathbf{s}$ \\
\hline
\end{tabular}

Notas: DNEC= Dirección Nacional de Estadísticas y Censos; DNT= Departamento Nacional de Traba jo; IS= Investigaciones Sociales; REA=Revista de Economia Argentina.

Fuente: elaboración propia.

El cuadro 1 sigue el argumento de Platt de manera estilizada. Demuestra que los estudios sobre Argentina hacen referencia a publicaciones oficiales, como las Investigaciones Sociales del Departamento Nacional de Trabajo (en adelante, DNT), o al propio DNT sin una referencia específica, así como al informe de 1963 de la Dirección Nacional de Estadísticas y Censos (en adelante, DNEC) (DNEC, 1963). Esta última es la publicación oficial del ICV con los datos de la encuesta de presupuesto familiar de 1960 y tiene una estimación para los años 1914 a

7 Los criterios de selección de los trabajos del cuadro 1 son que el mismo debe citar un ICV o, alternativamente, una estimación de salario real especificando la fuente del deflactor de precios, durante parte del período considerado en este trabajo. La lista no pretende ser exhaustiva. Reúne algunos de los trabajos más significativos, cubriendo diferentes disciplinas, con énfasis en la historia económica. 
1960. La serie de la DNEC corresponde al índice de largo plazo publicado en un informe del DNT de 1937, que se extiende entre 1913 y 1936 (RA-DNT, 1937: 20). Al contrario de lo que sostienen Harriague y Rayes (2018), ésta es la primer serie de largo plazo publicada oficialmente. Algunos autores citan los artículos de la REA y/o de Bunge. Bunge fue director de la REA, donde, como se mencionó anteriormente, se publicó el ICV por primera vez así como sus actualizaciones durante la década de 1920. En cuanto a los trabajos que hacen referencia a series no oficiales o a fuentes secundarias, los trabajos de della Paolera y Ortíz (1995), Díaz Alejandro (1981) e IEERAL (1986) son los citados con mayor frecuencia. Como muestra el cuadro, tanto della Paolera y Ortíz como IEERAL hacen referencia a Díaz Alejandro, quien, a su vez, cita la serie de la DNEC. ${ }^{8}$

La conclusión que surge del cuadro 1 es que, generalmente, los autores que analizan la historia argentina usan la misma estimación del costo de la vida. Javier Villanueva (1964) es la excepción, pero rara vez se hace referencia a su investigación. El cuadro 1 muestra cómo las estimaciones de Bunge se fusionaron con la estimación del DNT publicada en 1935 (RA-DNT, 1935), para luego viajar en el tiempo como ciencia ya constituida. ${ }^{9}$ Esta estimación, que de acuerdo con este trabajo tiene varios problemas, no es cuestionada y es utilizada sin el correspondiente análisis acerca de cómo se produjo. En algunos casos (Díaz Alejandro, 1981; Galiani y Gerchunoff, 2003; Salvatore, 2010; Sturzenegger y Moya, 2003; Williamson y Taylor, 1994, entre otros) el ICV de Bunge es empleado en modelos econométricos o estadísticos para probar diferentes hipótesis. De esta forma, "los pensamientos y los errores de una generación conducen demasiado fácilmente a las conclusiones de otra" (Platt, 1989: ix).

\section{De-construyendo el ICV de Bunge: su metodología según las publicaciones originales ${ }^{10}$}

La estimación de ICV se convirtió en una práctica generalizada a principios del siglo XX y Argentina no fue la excepción. El primer número de la REA, publicado en 1918, contiene un artículo de Bunge con una serie del ICV. Para ese entonces, Bunge se encontraba plenamente integrado al aparato estadístico nacional: entre 1913 y 1915 presidió la División de Estadística (en adelante, DE) del DNT y entre 1916-1921 y 1923-1925 fue el Director de la DGEN. La revista, fundada y dirigida por él, publicaba distintos tipos de artículos y era precursora en el análisis sobre estrategias de desarrollo y políticas económicas que pretendían generar el dinamismo perdido durante la Primera Guerra Mundial (Llach, 1985:52). Estaba financiada por publicidad oficial y privada, y sus investigaciones se basaban en estadísticas públicas y privadas (Pantaleón, 2004: 185). El ICV era parte de la

8 della Paolera y Ortíz también citan a la REA y otro trabajo de della Paolera (1988) para los años previos a 1910.

9 La estimación del DNT también tiene una serie de problemas que son analizados en Lanata Briones (2016 y en prensa b).

10 Para una explicación más detallada de esta etapa ver Lanata Briones (2016). 
visión macroeconómica de Bunge, que también se fundamentaba sobre la base de estimaciones de ingreso nacional, las estadísticas del comercio exterior y las encuestas de recursos y gastos de la familia obrera, todas estadísticas elaboradas por Bunge (González Bollo, 2014: 141-59). Esta caja de herramientas estadísticas, entendida como conocimiento objetivo y neutral, tenía como fin ser el sustento empírico de su postura. La visión macroeconómica de Bunge - que resaltaba la importancia del mercado interno como consumidor de producción manufacturera de origen nacional - se distanciaba del consenso ortodoxo existente que creía que la economía argentina retornaría a la dinámica agroexportadora previa a la Primera Guerra Mundial. ${ }^{11}$ La política industrial debía ser una política económica autónoma (Crovetto \& Zeolla, 2019). Para Bunge, el ICV era la base para estimar el coeficiente de corrección monetaria, el cuantificador del poder de compra del peso moneda nacional (Bunge, 1920a).

El ICV se publicó por primera vez de manera privada a pesar de estimarse en base a información generada por el aparato estadístico nacional. Las cifras del artículo de la REA de 1918 fueron actualizadas en 1919 (Bunge, 1919) y 1920 (Valle y Ferrari, 1920). En 1924, la DGEN publicó el ICV, convirtiéndolo en una estadística pública. La última actualización de esta serie (hasta 1926) también fue parte de la REA (Bunge, 1928). Para entender la metodología del índice, es necesario deconstruirlo analizando los primeros cuatro reportes mencionados. Como Bunge tuvo un rol central en los mismos, existe una asociación clara y estrecha entre Bunge y el ICV entre 1918 y 1924 que condicionó la trayectoria del indicador (Lanata Briones, en prensa a). Esta vinculación se deduce no sólo de su firma y participación en los cuatro reportes sino también de su rol en la introducción al sistema estadístico argentino de los números índices. ${ }^{12}$ Por ello, los reportes de la REA de 1918 y 1919 describen detalladamente cómo estimar números índices. Esta nueva herramienta estadística debía ser claramente explicada, entendida y por ende legitimada. La introducción de estos artículos es en una "guía metodológica y teórica, la garantía de precisión y verdad” (Scott, 1988: 124). La explicación pretendía resaltar la objetividad y neutralidad del índice, demostrar dominio sobre la metodología y proporcionarle al ICV un perfil científico.

Bunge comienza la explicación sobre la estimación del ICV con el análisis de la estructura de gasto, basándose en las encuestas de 1913 y 1914 que contenían información sobre 377 familias obreras de distintos barrios de la Ciudad de Buenos Aires, inicialmente publicadas en los Anuarios estadísticos del trabajo (RABDNT, 1915; RA-BDNT, 1916). En base a estas encuestas, Bunge estimó que el 42\% del salario se destinaba a la compra de alimentos, $19 \%$ al pago de alquileres, $31 \%$ se utilizaba en otros gastos y el $8 \%$ se ahorraba. Los ICV no consideran el ahorro. Por ello Bunge se deshizo de este componente, reasignando las proporciones de la siguiente forma: 50\% para alimentación, 20\% para alquiler y el restante 30\%

11 Asiain (2014) analiza detalladamente las ideas económicas de Bunge.

12 Los índices del comercio exterior fueron publicados en 1917 -antes del ICV- en El intercambio económico de la República Argentina en 1916. Tales indicadores introdujeron los números índices al sistema estadístico argentino, al contrario de lo que sostiene Pantaleón (2004: 190), que sitúa dicha introducción con el ICV. Sin embargo, el reporte sobre comercio exterior no posee explicación metodológica alguna acerca de cómo estimar números índices. 
para vestido, electricidad y otros gastos. ${ }^{13}$ Estos componentes se convirtieron en los tres capítulos del ICV. ${ }^{14} \mathrm{El}$ índice se estimaba anualmente como promedio ponderado de los tres capítulos. Para validar este procedimiento, Bunge comparó estas tres proporciones con datos de otros países y sostuvo que "teniendo en cuenta la equivalencia que se observa en todas partes en la distribución de los gastos cuando se les agrupa en aquellos tres rubros, podemos adoptar la referida distribución para todo el país” (Bunge, 1918: 43). Esta “equivalencia”, para Bunge, también implicaba que los datos de la Ciudad de Buenos Aires podían extenderse a la totalidad de la Argentina.

Según Bunge, carne y pan eran los alimentos más consumidos por los hogares argentinos en aquel entonces. Cada uno concentraba un 30\% del gasto en alimentos y el restante $40 \%$ se destinaba a otros alimentos. El capítulo alimentos era el promedio ponderado de esos tres grupos. El agregado carne era un promedio ponderado del precio de la carne vacuna (80\%), ovina (15\%) y porcina (5\%). El componente otros alimentos era un promedio simple de los precios de catorce bienes. ${ }^{15}$ Los precios utilizados para estimar los bienes del capítulo alimentos eran precios mayoristas de la Ciudad de Buenos Aires (Valle y Ferrari, 1920:254). Es fácil contrastar que esos precios son los mismos que los publicados en Intercambio económico de la República, el reporte que contenía los índices del comercio exterior (DGEN, 1918: 158-67). Bunge creía que el tipo de precios utilizados no era relevante porque, para él, la relación entre precios minoristas y mayoristas era casi constante (DGEN, 1924: 12).

Según Bunge, el DNT recolectaba datos sobre el precio del alquiler, y los diferentes reportes presentan números índices con dicho precio, que son los utilizados para estimar el ICV. Ningún reporte especifica si dicho precio corresponde al alquiler de una habitación, de un departamento o de una casa, ni detalla las características de la unidad habitacional.

Por último, respecto al capítulo otros gastos, Bunge explicó que "las variaciones de precios de los artículos de lana, algodón y otros textiles (excluida la seda), herramientas, enseres, kerosene y demás artículos que forman este rubro, están reguladas por las oscilaciones de los precios de los artículos importados que, además de representar una buena parte de la totalidad del consumo, dan lugar a oscilaciones correlativas en el mercado interno" (Bunge, 1918: 53). Al igual que con el capítulo alquiler, Bunge solamente publicó el valor agregado del mismo en cada uno de los cuatro reportes analizados.

Si bien esta metodología es, a grandes rasgos, la base de la estimación del ICV de Bunge, algunas diferencias existen entre las cuatro publicaciones. En el artículo de la REA de 1919 Bunge cambió las ponderaciones de los capítulos dado el incremento del 30\% del precio del alquiler que, según Bunge, no fue acompañado

13 De ahora en adelante, dicho componente se denominará otros gastos.

14 El término capítulo no fue utilizado por Bunge.

15 Aceite, arroz, azúcar, leche, carbón, leña, café, té, yerba, harina, grasas, papas, vino y tabaco. 
por un aumento salarial. Argumentado que los trabajadores no podían modificar su situación habitacional porque generalmente las familias vivían en una habitación, Bunge infirió un cambio en el patrón de consumo, aumentando la participación del capítulo alquiler al 26\% y reduciendo la de otros gastos al 24\%. $\mathrm{Al}$ analizar en detalle los números publicados en los diversos artículos, surge que en la estimación del ICV esa nueva escala de ponderaciones se utiliza sin realizar el empalme correspondiente. Es decir, para los valores hasta 1918 o 1919 inclusive ${ }^{16}$ Bunge estima el ICV con las ponderaciones 50-20-30, mientras que para los años siguientes se utilizan las proporciones 50-26-24 sin empalmar las dos estimaciones. En el artículo de la DGEN, el número de bienes considerados en el componente otros alimentos se incrementó a 18 ítems. ${ }^{17}$

\section{Construyendo el ICV de Bunge: sus problemas}

De los cuatro artículos anteriormente descriptos se infiere que el ICV de Bunge está principalmente basado en precios en lugar de cantidades. Un proxy de cantidades sólo se aplicó en las participaciones de los tres capítulos del índice y en algunos artículos dentro del capítulo alimentos. Sin embargo, no se sabe de dónde Bunge obtuvo las preferencias de consumo de los alimentos. El capítulo alimentos se actualizó utilizando precios mayoristas. Ningún reporte contiene especificaciones respecto al tipo de vivienda y a los precios considerados para estimar el capítulo alquiler. La participación del mismo experimentó un cambio que no fue introducido en la serie realizando un empalme apropiado. El capítulo otros gastos se estimó utilizando información sobre importaciones. Asimismo, en los artículos de la REA y de la DGEN se hizo gran hincapié sobre la explicación de la metodología de los números índices detrás de las estimaciones. Bunge proporcionó poca información sobre las características del hogar que el índice debía representar. Si bien claramente buscaba reflejar a la clase trabajadora de la Ciudad de Buenos Aires, su objetivo de caracterizar a la familia obrera promedio es implícito y debe ser inferido, tal como se explica a continuación. Dado que las encuestas de gastos de 1913 y 1914 se llevaron a cabo entre los trabajadores de la Ciudad de Buenos Aires, sumado a la afirmación de Bunge de que los precios se movían de manera similar en todo el país, se infiere que el ICV se refería a dicha población urbana. Para Bunge, concentrarse en los trabajadores significaba centrarse en "la mayor parte del consumo general” (Bunge, 1918: 47).

Los Anuarios estadísticos del trabajo, compilados cuando Bunge estaba a cargo de la DE del DNT, contenían información sobre las encuestas de gasto de 1913 y 1914 a familias obreras de la Ciudad de Buenos Aires. Por ende, Bunge conocía su contenido, el procedimiento metodológico y los datos obtenidos. Los Anuarios no especificaban cómo se recopiló la información, qué técnica de muestreo se utilizó

16 Esto depende del artículo en cuestión. En los artículos de 1919 y 1920 de la REA, el cambio de proporciones se realiza en 1918; para la publicación de la DGEN esto ocurre en 1919.

$17 \mathrm{~A}$ los bienes considerados anteriormente se le sumó queso, huevos, pescado y manteca. 
o cómo se llevaron a cabo las encuestas. Sin embargo, contenían una copia del cuestionario utilizado. A las familias se les preguntó cuánto dinero habían gastado mensualmente y anualmente en categorías predeterminadas ${ }^{18}$ sin que tuvieran que detallar los bienes alimenticios comprados. Dado el número de categorías y la disponibilidad de precios oficiales, el ICV podría haber sido construido de manera diferente. Los combustibles y la luz podrían haber formado parte de un cuarto capítulo, en lugar de que el carbón y la leña estén en el capítulo alimentos y el kerosene en el de otros gastos. El tabaco podría haber sido excluido del capítulo alimentos, mientras que el vino podría haber sido un componente separado dentro del mismo, como la carne y el pan. Sin embargo, como se explicó anteriormente, en los Anuarios los resultados de los componentes del gasto se dividieron sólo en alimentos, alquiler, ahorro y otros gastos. Tampoco existe en ellos indicación alguna de cómo y por qué se determinaron estas cuatro categorías. Como alimentación era una categoría única en los cuestionarios, los Anuarios no podrían haber sido la fuente de Bunge para establecer los componentes del capítulo alimentos. Por ende, no hay explicación ni evidencia detrás de la elección de ese listado de alimentos. Dado el cuestionario, la falta de desagregación en los datos publicados y utilizados, sugiere la existencia de problemas con la información de las encuestas, ya que la presentación de datos altamente agregada es una forma de minimizar potenciales errores que podrían socavar las estimaciones de Bunge. Esta inexactitud se infiere también de la declaración de la DE del DNT respecto de la falta de colaboración e interés de los trabajadores en las encuestas de gasto realizadas durante la década de 1920 (RA-CMDNT, 1926; RA-CMDNT, 1927), lo cual sugiere una desconfianza general hacia los números oficiales, especialmente por parte de la clase obrera.

Bunge obtuvo el valor del salario promedio anual de los trabajadores de la Ciudad de Buenos Aires, equivalente a m\$n1.814,5, realizando el promedio ponderado de los valores de 1913 y 1914, en base al número de casos encuestados. Sin embargo, no utilizó ese mismo procedimiento para estimar las proporciones de los cuatro componentes del gasto, que serían de 43,8\% para alimentos, 18,7\% para alquiler, $30,3 \%$ para otros gastos, y 7,2\% para ahorro. ${ }^{19}$ La distancia entre estos valores y los de Bunge no es significativa, pero es importante resaltar la inconsistencia en su procedimiento y la falta de transparencia en su explicación. Asimismo, en base a esto se infiere que el ICV se refería a la familia obrera promedio.

El ICV de Bunge fue estimado utilizando precios mayoristas. Sin presentar la evidencia correspondiente, Bunge sostuvo que "tratándose de estudiar las "oscilaciones" del costo general de la alimentación, es suficiente conocer las del pan, la carne y estos artículos, al por mayor, dada la correlación que existe entre esas oscilaciones cuando abarcan periodos anuales, y las de los precios al por menor y los que "paga el obrero'”' (1918:48). Este procedimiento sigue la sugerencia de Irving Fisher sobre el uso de precios mayoristas, haciendo caso omiso a la de Arthur Bowley, que favorecía

18 Alimentación, vestidos, alquiler, combustibles, luz, impuestos, seguro de vida, muebles y utensilios, libros y periódicos, escuelas particulares, transporte, maestros particulares, culto religioso, caridad y beneficencia, sociedades obreras, enfermedades, diversiones, vinos y licores, tabaco, otros gastos.

$19 \mathrm{Al}$ omitir el ahorro, esas proporciones se transforman en $47,2 \%, 20,1 \%$ y $32,7 \%$ respectivamente. 
el uso de precios minoristas (Sember, 2013: 376). Al margen de esta observación, lo importante es que Bunge no tomó la decisión por la falta de datos, ya que la DE del DNT recolectaba precios minoristas hacía varios años (ver, por ejemplo, RA-BDNT, 1918; RA-CMDNT, 1922). Sin embargo, podría pensarse que Bunge utilizó precios mayoristas por comodidad pues eran compilados y publicados en sus reportes sobre comercio exterior. También podría pensarse que en base al listado de precios mayoristas publicado en Intercambio económico de la República con datos para el período 1910-1917 (DGEN, 1918: 158-67), Bunge seleccionó los bienes que formarían parte del capítulo alimentos. Los gráficos 1 y 2 muestran las variaciones anuales de los precios mayoristas y minoristas de la carne y el pan.

Gráfico 1. Variación porcentual anual del precio de la carne, 1911-1920

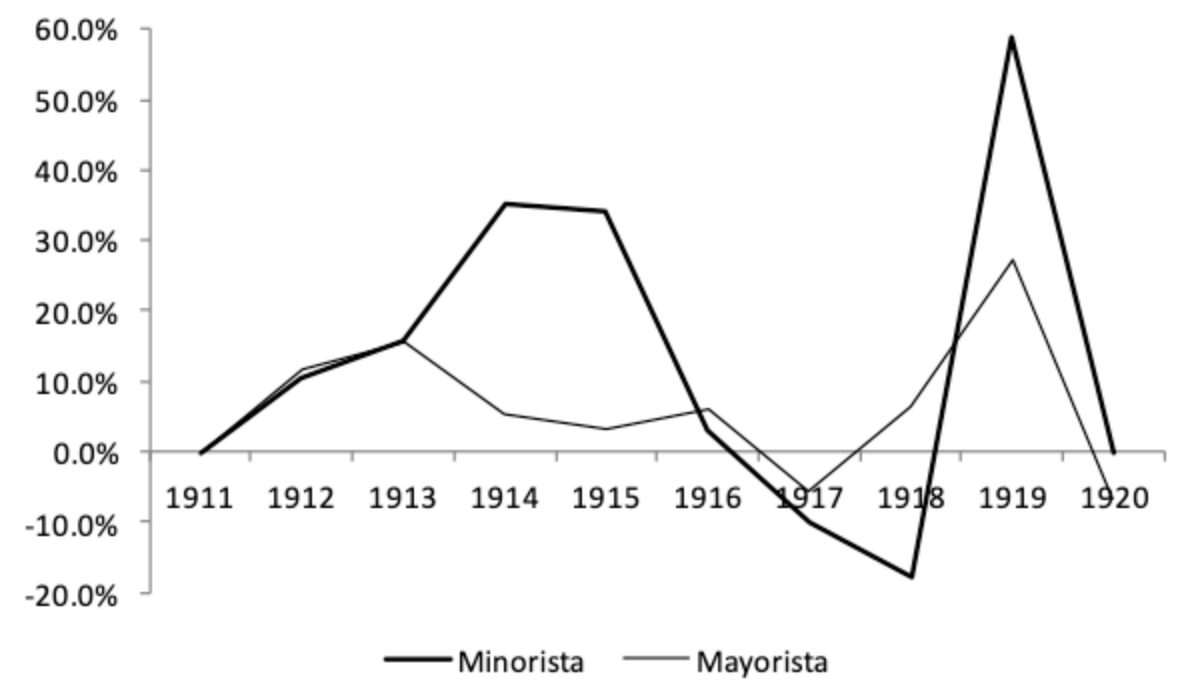

Fuentes: elaboración propia en base a Bunge (1918); Di Tella (1964); DGEN (1924).

Gráfico 2. Variación porcentual anual del precio del pan, 1911-1920

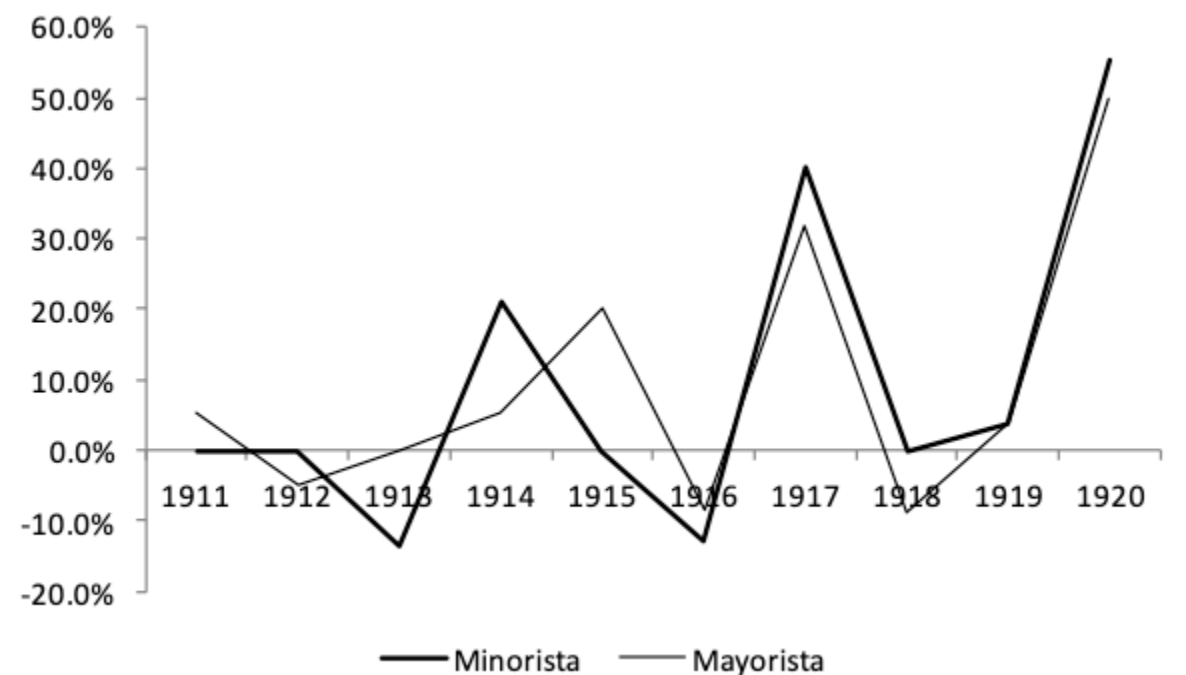

Fuentes: elaboración propia en base a Bunge (1918); Di Tella (1964); DGEN (1924). 
A pesar de las similitudes, especialmente en el caso del pan, los precios mayoristas y minoristas no presentaron la misma tendencia entre 1911 y 1920. Generalmente, los precios minoristas fluctuaron más que su equivalente mayorista, socavando la hipótesis de Bunge y Fisher de una correlación entre los precios al por menor y al por mayor. El uso de precios mayoristas no reflejaba la situación de los trabajadores quienes seguramente pagaban por estos bienes al valor minorista. Los gráficos 1 y 2 muestran que el uso de precios mayoristas distorsionarían los resultados del ICV de Bunge.

Bunge también afirmó que si bien el valor absoluto inicial de la canasta difería para la Argentina en su conjunto y para la Ciudad de Buenos Aires, debido a los diferentes patrones de consumo, las variaciones anuales de los precios eran las mismas en toda la República. En enero de 1921 la REA publicó un cuadro, reproducido aquí como el cuadro 2, con números índices calculados para ocho alimentos de consumo básico en diferentes ciudades argentinas entre enero de 1919 y junio de $1920 .{ }^{20}$

Cuadro 2. Evolución de los precios de alimentos en diferentes ciudades argentinas, enero 1919-junio 1920. Base: Enero 1919=100

\begin{tabular}{l|cccc}
\hline & Enero 1919 & Junio 1919 & Enero 1920 & Junio 1920 \\
\hline S. M. de Tucumán (TU) & 100 & 129 & 137 & 169 \\
Las Flores (BA) & 100 & 106 & 117 & 154 \\
Salta (SA) & 100 & 103 & 140 & 152 \\
San Juan (SJ) & 100 & 116 & 128 & 149 \\
Azul (BA) & 100 & 105 & 136 & 141 \\
Mendoza (ME) & 100 & 110 & 110 & 140 \\
Rosario (SF) & 100 & 113 & 115 & 139 \\
Mercedes (BA) & 100 & 108 & 123 & 138 \\
Concordia (ER) & 100 & 102 & 126 & 134 \\
Luján (BA) & 100 & 108 & 114 & 133 \\
Santiago del Estero (SE) & 100 & 98 & 116 & 133 \\
Córdoba (CO) & 100 & 106 & 112 & 133 \\
Paraná (ER) & 100 & 98 & 110 & 129 \\
San Luis (SL) & 100 & 107 & 111 & 119 \\
Corrientes (COR) & 100 & 93 & 106 & 117 \\
Buenos Aires (BA) & 100 & 104 & $\mathbf{1 1 0}$ & $\mathbf{1 1 6}$ \\
Tandil (BA) & 100 & 109 & 109 & 115 \\
La Rioja (LR) & 100 & 107 & 111 & 114 \\
Bahía Blanca (BA) & 100 & 102 & 93 & 102 \\
\hline
\end{tabular}

Fuente: REA (1921).

Entre enero de 1919 y junio de 1920, los precios fluctuaron de manera muy desigual entre las diferentes ciudades, tal como muestra el cuadro 2. De las 19 localidades consideradas, la Ciudad de Buenos Aires se situaba en el puesto 16, con un incremento del

20 Los artículos de primera necesidad incluían pan, carne, azúcar, arroz, papas, pasta, aceite y yerba. El reporte no explica cómo se calcularon los números índices. 
16\% en el período, muy por debajo del aumento del 69\% en San Miguel de Tucumán. Incluso dentro de la provincia de Buenos Aires, por ejemplo, la dispersión fue alta. Mientras que Las Flores experimentó el segundo incremento más sustancial de la muestra, Bahía Blanca fue la ciudad con el menor aumento. Al menos durante este período, la hipótesis de Bunge de que las fluctuaciones del ICV eran representativas para Argentina en su conjunto no se corrobora. Si la Ciudad de Buenos Aires experimentó sistemáticamente incrementos de precios menores que el resto del país, el ICV de Bunge habría subestimado las fluctuaciones de precios para otras localidades.

En base a la información provista por Bunge en los cuatro artículos considerados, se puede inferir que los movimientos del capítulo alquiler estaban basados en todos los tipos de alquileres y arrendamientos del país. Esta afirmación se fundamenta en el hecho de que cuando Bunge explicó el costo general de todos los gastos argentinos en el artículo de la REA de 1918, argumentó que él mismo había estimado el valor de todos los alquileres y arrendamientos en Argentina para 1914. Sobre este número después calculó "la subida o baja relativa a ese año, dado que la superficie explotada poco ha aumentado durante estos años y que la edificación está poco menos que paralizada" (1918: 60-1). Asimismo aclaró que el DNT tenía datos para el período 1912 a 1915 y que los restantes años habían sido estimados por él. La serie publicada en el apartado sobre el costo general es exactamente la misma que la del capítulo alquiler. El gráfico 3 muestra la variación anual del precio del alquiler según el artículo de la REA de 1918 y la publicación de la DGEN de 1924 (la serie denominada oficial en el gráfico) y el precio promedio de una habitación, una habitación en un departamento y una habitación en una casa. Las últimas tres series fueron publicadas en la Revista de Economía y Estadística y se refieren a los valores de la Ciudad de Buenos Aires. Mientras que las tres series del Instituto de Estadística (en adelante,IE) tienen variaciones relativamente similares, el capítulo alquiler se comportó de manera diferente, como se observa en el gráfico 3.

Gráfico 3. Variación porcentual anual del precio del alquiler según diferentes fuentes, 1913-1923

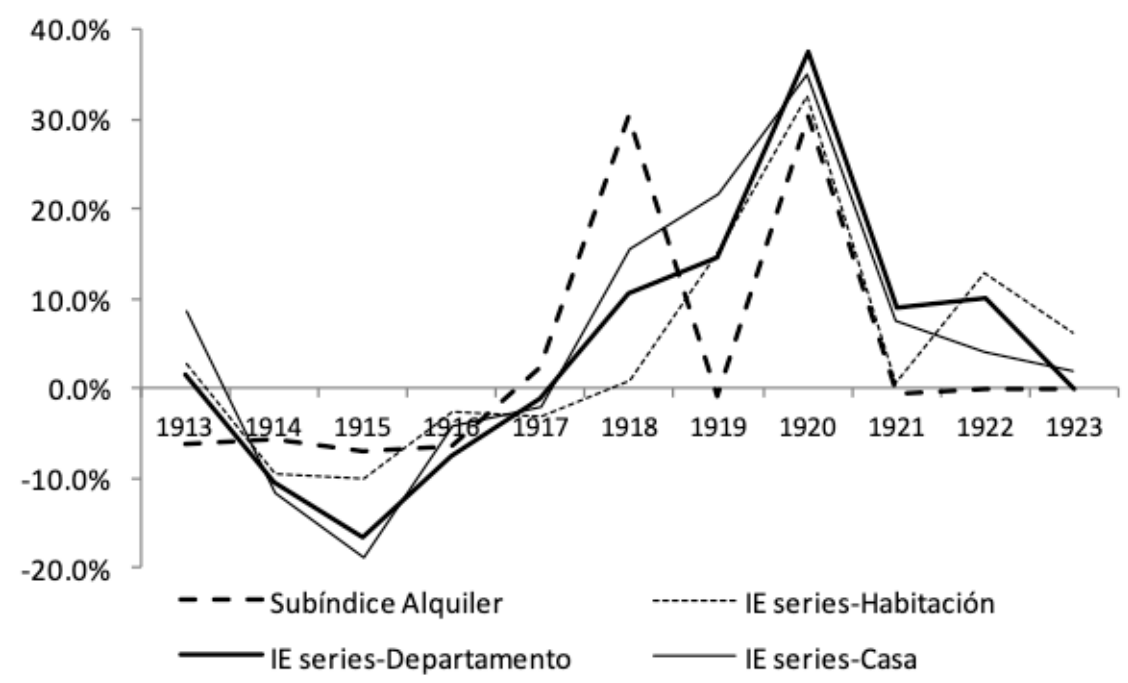

Fuentes: elaboración propia en base a Bunge (1918); DGEN (1924); IE, (1939).

Nota: 'Habitación' se refiere al precio promedio de un cuarto, 'Departamento' se refiere al precio promedio de una habitación en un departamento, y 'Casa' se refiere al precio promedio de una habitación en una casa. 
En 1918, el incremento del 30\% en el capítulo alquiler, que Bunge utilizó para justificar el cambio en las proporciones de los capítulos del ICV, fue de la mitad al considerar el alquiler de una habitación dentro de una casa y del 0,9\% para una habitación. Según los datos del IE, los alquileres se incrementaron mucho más en 1919, pero por sobre todo en 1920. Dicho año muestra una variación similar entre las cuatro series, con un aumento de entre el 30,4\% y el 37,5\%. El menor incremento corresponde al capítulo alquiler de Bunge. Según el IE, sus estimaciones eran similares a las del DNT para el período 1933-1938, pero en el artículo correspondiente nunca se mencionan los datos del período del ICV de Bunge. A pesar de los picos en 1918 y 1920, el capítulo alquiler fluctuó menos que los números compilados por el IE, que probablemente estaban más cerca de lo que pagaban los trabajadores ya que dichas series se generaban utilizando información de anuncios periodísticos. El uso de estos datos generaría un ICV menor al experimentado por los trabajadores. El gráfico 3 es otra evidencia de que el capítulo alquiler refleja las tendencias del país en su conjunto, debilitando la estimación oficial.

En base al incremento en 1918 del precio del alquiler de la serie oficial, Bunge modificó las proporciones de los tres capítulos del ICV de 50-20-30 a 50-26-24, sin brindar ningún tipo de evidencia. El cuadro 3 sintetiza los resultados de las encuestas de gasto categorizados según los tres capítulos del ICV. Dichas encuestas fueron realizadas por la DE del DNT siguiendo la metodología diseñada por Bunge y utilizada por él para determinar la estructura del ICV.

Cuadro 3. Estructura porcentual de gasto de la clase trabajadora, 1913-1929

\begin{tabular}{l|cccccccccccc}
\hline & 1913 & 1914 & $\mathbf{1 9 1 9}$ & $\mathbf{1 9 2 2}$ & $\mathbf{1 9 2 3}$ & $\mathbf{1 9 2 4}$ & $\mathbf{1 9 2 5}$ & $\mathbf{1 9 2 6}$ & $\mathbf{1 9 2 8}$ & $\mathbf{1 9 2 9}$ & $\begin{array}{c}\text { Promedio } \\
\mathbf{1 9 1 3 - 2 3}\end{array}$ & $\begin{array}{c}\text { Promedio } \\
\mathbf{1 9 1 3}-29\end{array}$ \\
\hline Alimentos & $47.9 \%$ & $46.1 \%$ & $59.6 \%$ & $55.0 \%$ & $53.0 \%$ & $55.0 \%$ & $55.0 \%$ & $48.0 \%$ & $51.5 \%$ & $51.0 \%$ & $52.3 \%$ & $52.2 \%$ \\
Alquiler & $20.0 \%$ & $20.3 \%$ & $17.8 \%$ & $18.0 \%$ & $17.0 \%$ & $18.0 \%$ & $18.0 \%$ & $22.0 \%$ & $22.5 \%$ & $20.0 \%$ & $18.6 \%$ & $19.4 \%$ \\
Otros gastos & $32.1 \%$ & $33.6 \%$ & $\mathbf{2 2 . 6 \%}$ & $\mathbf{2 7 . 0 \%}$ & $\mathbf{3 0 . 0 \%}$ & $\mathbf{2 7 . 0 \%}$ & $\mathbf{2 7 . 0 \%}$ & $\mathbf{3 0 . 0 \%}$ & $\mathbf{2 6 . 0 \%}$ & $29.0 \%$ & $29.1 \%$ & $28.4 \%$ \\
\hline
\end{tabular}

Fuente: elaboración propia en base a RA-BDNT (1915); RA-BDNT (1916); RA-CMDNT (1920); RA-CMDNT (1927); RA-CMDNT (1929); RA-CMDNT (1930).

La afirmación de Bunge de que el aumento en 1918 en los alquileres generó un incremento en la participación de tal gasto a expensas de otros gastos no se verifica al examinar los números de 1919 y 1920 (cuadro 3), lo cual puede considerarse como otra prueba de que el capítulo alquiler no reflejaba lo pagado por los trabajadores de la Ciudad de Buenos Aires. El cuadro 3 muestra que la estructura de gasto de la clase trabajadora se mantuvo relativamente estable y similar a las estimaciones de 50-20-30 de Bunge para el período 1913 a 1923. El componente alquiler es el más estable de los tres y, en todo caso, los reemplazos por fluctuaciones de precios ocurrían entre el componente alimentos y el componente otros gastos, particularmente si comparamos un plazo de tiempo más largo, es decir 1913-1929. Esta evidencia socava la justificación de Bunge detrás de la variación de las proporciones de los capítulos.

Como se explicó anteriormente, la forma en que Bunge definió el capítulo otros gastos implica que este sólo comprendía bienes importados. En 1918 Bunge afirmó que dicho capítulo se estimaba con el valor importado, lo que significa que las 
cantidades y no sólo los precios de los bienes cambiaban cada año, mientras que las cantidades del capítulo alimentos, por ejemplo, permanecían fijas. Según Bunge, 21 números índices eran estimados anualmente utilizando grupos de artículos y bienes aislados como carbón, ya considerado en el capítulo alimentos, y "arpillera" (1918: 54). Bunge brindó pocos detalles de los 21 índices utilizados, lo que dificulta la reestimación del capítulo. Según su explicación, se construían los mismos números índice cada año, lo que significa que el país importaba sistemáticamente todos los productos, una suposición exagerada. Asimismo, se infiere que el capítulo otros gastos no siempre contenía los mismos bienes, alterando la naturaleza del ICV. Más importante aún, la verificación de diversas fuentes primarias muestra que -a pesar de la definición de Bunge del capítulo que sugiere que el mismo contenía principalmente artículos relacionados con la vestimenta- los números del capítulo otros gastos se referían a las importaciones argentinas totales y no sólo a un grupo seleccionado de bienes. Esto incluyó una lista de artículos muy diversos que potencialmente no fueron consumidos por los trabajadores, ni eran utilizados en la producción de indumentaria. De hecho, una comparación entre el capítulo otros gastos (Bunge, 1918: 54; Bunge, 1919: 203; DGEN, 1924: 21) y el índice oficial de precios de importación de la Argentina para los años 1910 a 1926 (Dirección Nacional de Investigaciones, Estadística y Censos, 1948:xv) muestra que ambas estimaciones tenían fluctuaciones interanuales prácticamente idénticas. Aunque se basó en la comprensión de Bunge de la dinámica económica argentina como importadora de bienes manufacturados (Lanata Briones, en prensa a), el capítulo otros gastos no reflejaba plenamente lo que pretendía describir.

Las decisiones de Bunge respecto a cómo estimar el ICV no fueron tomadas por falta de conocimiento o por ignorancia. A pesar de no ser estadístico de formación, Bunge conocía la literatura existente y tenía vínculos con académicos reconocidos internacionalmente. ${ }^{21}$ Durante sus estudios de ingeniería en Alemania, conoció los preceptos de la Escuela Histórica Alemana (Lucchini et al, 2000), una disciplina basada fuertemente en el uso de estadísticas. Las referencias de Bunge al trabajo de sus colegas, un elemento de persuasión que genera credibilidad (Latour, 1987: 33), son una prueba de su conocimiento. El ingeniero referenció "las investigaciones retrospectivas sobre salarios y costo de la vida de en el siglo XIX, realizadas por Sauerbeck, Wood y Levasseur y otros economistas, y por el "Board of Trade" de Inglaterra y "L’Office du Travaille” de Francia” (1918: 39), el trabajo realizado para los Estados Unidos Cost of Living and Retail Prices of Food, Recherches sur la théorie du prix de Dudoff Auspitz y Richard Sieben, ${ }^{22}$ The Purchasing Power of Money de Fisher ${ }^{23}$ y Elements of Statistics de Bowley. ${ }^{24}$ Los últimos dos libros formaban parte del programa del curso de estadística de la Facultad de Ciencias Económicas de la Universidad de Buenos Aires en el cual Bunge daba clases. ${ }^{25}$

21 Un análisis en profundidad del contexto y las ideas de Bunge respecto a la estimación del ICV se realiza en Lanata Briones (en prensa a).

22 González Bollo (2012: 17-22) explora otras influencias.

23 Fernández López (1994: 671) y Sember (2013: 379) analizan la relación entre Bunge y Fisher.

24 Los libros de Bowley y Fisher llevaron a Bunge a concebir el análisis de problemas mediante el uso de números índices (Fernández López, 1994: 667).

25 (1919). Estadística 1, Anales de la Facultad de Ciencias Económicas, pp. 605-06. 
También existió contacto personal entre Bunge y Bowley. Una carta de Bowley a Bunge fue transcrita en la REA y Bunge afirmó haber recibido de Bowley una copia de su presentación de abril de 1919 a la Royal Statistical Society de Londres. La biblioteca de la London School of Economics and Political Science posee una copia del libro de Bunge Los problemas económicos del presente con una inscripción del propio Bunge dirigida al "distinguido profesor Arthur L. Bowley", con fecha junio de 1920. Incluso cuando se distanció del sistema estadístico nacional después de 1925, Bunge y su trabajo continuaron siendo reconocidos internacionalmente. ${ }^{26}$ Por ejemplo, fue uno de los miembros fundadores del Instituto Inter-Americano de Estadísticas (Inter-American Statistical Institute) en 1940 (IASI, 1941: 822-51).

\section{Re-construyendo el ICV de Bunge}

Siguiendo la metodología de de-construcción/construcción/re-construcción de estadísticas, en los apartados precedentes se ha de-construido y construido el ICV de Bunge, quedando pendiente su re-construcción, que se lleva a cabo en esta sección, en la cual se presentan y examinan las series re-construidas del ICV de Bunge para el período 1912-1932. El objetivo es reproducir el ICV de Bunge lo más fielmente posible a su formato y concepción original, focalizándose en el efecto de las diferentes suposiciones. Las series re-construidas son comparadas con el índice oficial, es decir, con el usado por la historiografía, que usa el índice de Bunge, tal como se muestra en el cuadro 1. Dado que el núcleo de la explicación de las divergencias es la forma en que se re-construyen las estimaciones, el análisis no está fuertemente influenciado por el año base de la serie. Las estimaciones comienzan en 1912 porque la serie de precios de alquiler del IE, la fuente de este dato en las estimaciones re-construidas, arranca en ese año. Además, la existencia de precios minoristas oficiales es incompleta para 1910 y 1911. Terminan en 1932 ya que en 1935 el DNT lanza un nuevo ICV con base en octubre de 1933 (RA-DNT, 1935).

En casi todas sus publicaciones, Bunge presentó detalladamente los cálculos. Sobre la base de esa información y sus explicaciones metodológicas, se ha demostrado que Bunge no empalmó las series correctamente cuando decidió cambiar las proporciones de la estructura de gasto, ${ }^{27}$ que utilizó erróneamente precios mayoristas para los bienes alimenticios - a pesar de la disponibilidad de precios minoristas -, que el capítulo alquiler no reflejó el comportamiento de los precios de la Ciudad de Buenos Aires, y que la estructura de gasto de las familias obreras no fluctuó con los cambios en el precio del alquiler. Dadas las observaciones hechas respecto a estos cuatro aspectos, las dos re-construcciones del ICV de Bunge corrigen estas cuestiones hasta 1926. Las variaciones anuales del capítulo otros gastos, que reflejan los precios

26 Nueva evidencia del reconocimiento internacional de Bunge previo a su alejamiento del aparato estadístico nacional en 1925 puede encontrarse en Lanata Briones (en prensa a). Dicha evidencia es independiente de las menciones que el mismo Bunge hace de la misma y que son reproducidas por diversos autores, como Pantaleón (2004: 190) por citar un ejemplo.

27 Lanata Briones (2016: 116-117) muestra que, por esta falta de empalme y sin ajustar por ningún otro problema, para el intervalo $1910-1923$ el ICV de Bunge es 11,1\% menor que la serie empalmada correctamente. 
de las importaciones totales de Argentina, varían ligeramente entre las dos series re-construidas y la oficial utilizada por Bunge. Los números de esta última no se extienden después de 1926 y son utilizados hasta dicho año. Para el periodo 19271932, las dos series reconstruidas se actualizan en base a las variaciones del ICV en su totalidad calculado por Bunge (Bunge, 1940: 200). ${ }^{28}$ Las series re-construidas del ICV de Bunge se presentan en el gráfico 4 conjuntamente con el índice oficial. El cuadro 4 sintetiza las características de las tres series de dicho gráfico.

Cuadro 4. Diferencias principales entre las diferentes re-construcciones del ICV de Bunge

\begin{tabular}{|c|c|c|c|c|c|}
\hline $\begin{array}{c}\text { Nombre de la } \\
\text { serie }\end{array}$ & $\begin{array}{l}\text { Precios del } \\
\text { capítulo } \\
\text { alimentos }\end{array}$ & $\begin{array}{c}\text { Precios del capítulo } \\
\text { alquiler }\end{array}$ & $\begin{array}{c}\text { Precios del capítulo } \\
\text { otros gastos }\end{array}$ & $\begin{array}{l}\text { Hay empalme de } \\
\text { las series tras el } \\
\text { cambio de } \\
\text { participaciones? }\end{array}$ & $\begin{array}{l}\text { Estructura de } \\
\text { los capítulos }\end{array}$ \\
\hline $\begin{array}{l}\text { ICV re- } \\
\text { construido con } \\
\text { cambio }\end{array}$ & \begin{tabular}{|c} 
Minoristas para \\
1912-1926. \\
Variaciones del \\
ICV general \\
para 1927-1932 \\
\end{tabular} & $\begin{array}{l}\text { Habitación en la Ciudad } \\
\text { de Buenos Aires (serie } \\
\text { del IE) para 1912-1926. } \\
\text { Variaciones del ICV } \\
\text { general para 1927-1932 }\end{array}$ & $\begin{array}{c}\text { Estimaciones de } \\
\text { Bunge/REA para 1912- } \\
\text { 1926. Variaciones del } \\
\text { ICV general para 1927- } \\
1932 \\
\end{array}$ & $\mathrm{Si}$ & $\begin{array}{l}\text { 50-20-30 para } \\
1912-1918 ; 50- \\
24-26 \text { para } 1919- \\
1932\end{array}$ \\
\hline $\begin{array}{c}\text { ICV re- } \\
\text { construido sin } \\
\text { cambio }\end{array}$ & $\begin{array}{c}\text { Minoristas para } \\
\text { 1912-1926. } \\
\text { Variaciones del } \\
\text { ICV general } \\
\text { para 1927-1932 }\end{array}$ & $\begin{array}{l}\text { Habitación en la Ciudad } \\
\text { de Buenos Aires (serie } \\
\text { del IE) para 1912-1926. } \\
\text { Variaciones del ICV } \\
\text { general para 1927-1932 }\end{array}$ & $\begin{array}{c}\text { Estimaciones de } \\
\text { Bunge/REA para 1912- } \\
\text { 1926. Variaciones del } \\
\text { ICV general para 1927 } \\
1932 \\
\end{array}$ & $\mathrm{Si}$ & $\begin{array}{c}\text { 50-20-30 para } \\
1912-1932\end{array}$ \\
\hline ICV oficial & Mayoristas & $\begin{array}{c}\text { No la Ciudad de Buenos } \\
\text { Aires, probablemente } \\
\text { no una habitación (serie } \\
\text { de Bunge) }\end{array}$ & $\begin{array}{c}\text { Estimaciones de } \\
\text { Bunge }\end{array}$ & No & $\begin{array}{c}\text { 50-20-30 para } \\
1912-1918 ; 50- \\
24-26 \text { para } 1919- \\
1932\end{array}$ \\
\hline
\end{tabular}

Nota: En la estructura de los capítulos, los números se refieren a alimentos-alquiler-otros gastos.

Fuente: elaboración propia.

Gráfico 4. Estimaciones re-construidas del ICV de Bunge, 1912-1932. Base: 1912=100.

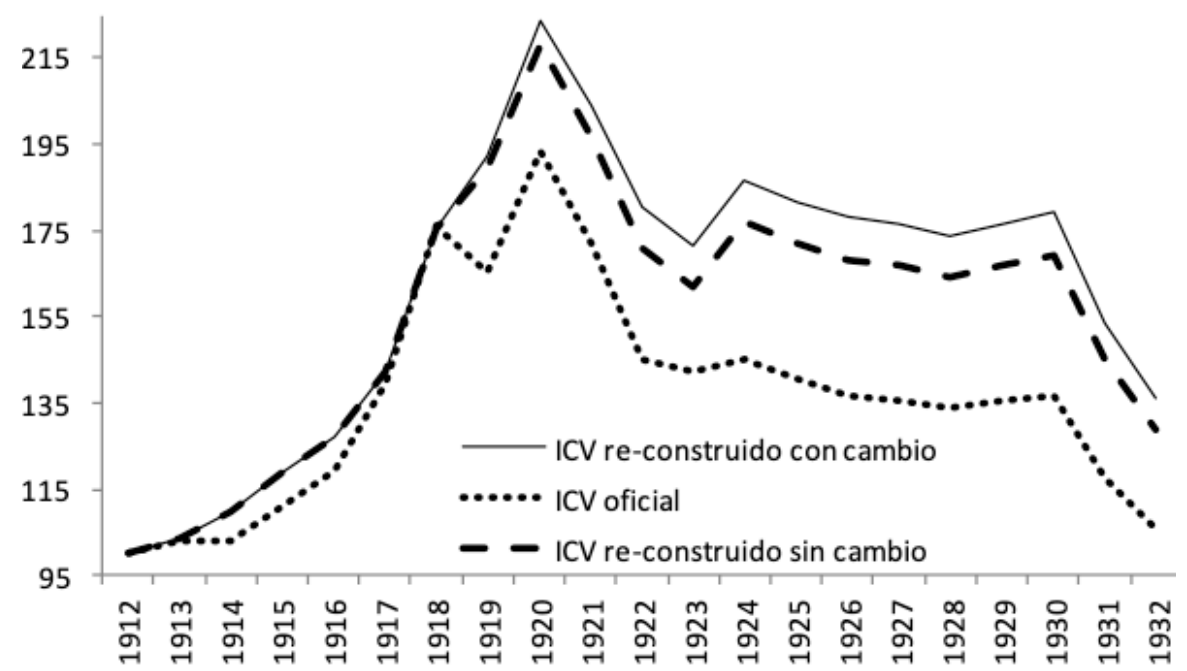

Fuente: elaboración propia. Ver Apéndice.

28 Lanata Briones (2016) propone otras dos re-construcciones del ICV de Bunge donde los procedimientos utilizados aquí para los capítulos alimentación y alquiler se extienden hasta 1932 y para el capítulo otros gastos se utiliza la serie de precios de importación de la Dirección Nacional de Investigaciones, Estadística y Censos (1948). Los números finales difieren mínimamente, pero las conclusiones son las mismas. 
Entre 1912 y 1918, ambas series re-construidas del ICV de Bunge son idénticas. A partir de 1919, siguiendo la metodología de Bunge, el ICV re-construido con cambio presenta una alteración en las participaciones de los capítulos, del 50-20-30 para alimentos, alquiler y otros gastos, respetivamente, al 50-24-26, pero empalmado correctamente. El ICV re-construido sin cambio mantiene las proporciones 50-2030 dada la evidencia presentada en el cuadro 3. Esa es la única diferencia entre las dos series re-construidas del ICV de Bunge y explica su divergencia después de 1918. Ambas usan los datos del precio del alquiler del IE, los mismos precios al por menor y el mismo índice de precios de importación para re-construir el capítulo otros gastos hasta 1926. Con la excepción de 1918, los índices re-construidos siempre exceden al ICV oficial, tal como se ve en el gráfico 4. El ICV oficial aumenta un 5,6\% entre 1912 y 1932, mientras que las series re-construidas se incrementan mucho más: el ICV re-construido sin cambio aumenta un 28,7\% y el ICV re-construido con cambio un 36,2\%.

Las tres series se mueven de manera parecida hasta 1918. Las diferencias entre las series re-construidas del ICV de Bunge y la serie oficial oscilan entre 0,5 puntos en 1913 y 8,2 en 1916. Dado que para esos años el capítulo otros gastos es el mismo y que las proporciones de los capítulos es la misma para las tres estimaciones, las diferencias provienen de los capítulos alquiler y alimentos. Las discrepancias muestran que hasta 1916, los precios al por menor de alimentos y / o el alquiler de una habitación en la Ciudad de Buenos Aires aumentaron más bruscamente que su equivalente mayorista o el capítulo oficial de alquiler. Ocurre lo contrario en 1917 y 1918 cuando la serie oficial alcanzó las estimaciones re-construidas del ICV de Bunge. En 1919, el ICV oficial declina un 6,2\% mientras que el ICV re-construido con cambio aumenta un 9,7\% y el ICV re-construido sin cambio un $8 \%$, generando una brecha de 27,1 puntos entre la estimación oficial y el primer ICV re-construido y una diferencia de 24,1 puntos al compararse con el segundo ICV re-construido. Las diversas trayectorias entre los ICV re-construidos y el oficial se deben a la metodología de empalme que se utiliza en las re-construcciones. A partir de 1919, la discrepancia se amplía, alcanzando en 1924 un valor de 42 puntos entre el ICV oficial y re-construido con cambio y de 32,2 puntos cuando el índice oficial se compara con el re-construido sin cambio. Entre 1924 y 1930, el gráfico 4 muestra una disminución estable de la brecha entre las tres estimaciones, especialmente entre los ICV re-construidos. El drástico descenso entre 1930 y 1932 se debe a la baja en los precios internacionales consecuencia de la Gran Depresión.

Algunos años son cruciales para la comparación de los ICV de Bunge re-construidos y el oficial. En 1924, el ICV re-construido con cambios aumenta un $9 \%$ y el ICV re-construido sin cambios un 9,2\%, mientras que el índice oficial aumenta sólo un 1,9\%. Como sucede en años anteriores, las discrepancias se deben tanto al comportamiento de los capítulos alimentos y alquiler como a las participaciones de los capítulos alquiler y otros gastos. Con respecto a los ICV re-construidos, el precio del alquiler de una habitación en la Ciudad de Buenos Aires disminuye un $4,5 \%$ dicho año. El precio minorista de la carne aumenta un 39\% y el del pan un $12,9 \%$. El componente otros alimentos se incrementa un 17\%. En las series reconstruidas, el capítulo alimentos aumenta un 22,2\%. En el ICV oficial, el capítulo 
alquiler no varía y el de alimentos se incrementa un 2,8\%. Las discrepancias en los valores de los capítulos alimentos y alquiler y el impacto diferencial de los capítulos alquiler y otros gastos debido a la alteración de su participación muestran cómo el uso de los precios minoristas en lugar de los precios al por mayor y los cambios en las participaciones producen estimaciones diferentes.

La caída en 1919 de la serie oficial se debe a la falta de empalme en dicha estimación tras la decisión de Bunge de cambiar las participaciones de los capítulos alquiler y otros gastos. Esta alteración, como se ha demostrado en la sección anterior, no posee fundamento empírico. Al empalmar la serie, los dos ICV reconstruidos aumentan entre 1918 y 1919 en lugar de disminuir, como sucede con la serie oficial, generando una brecha que nunca se cierra entre los dos grupos de series. Asimismo, dado que el valor oficial de 1920 se estimó siguiendo el procedimiento de 1919, el aumento anual de 1920 en la serie oficial es del 17,2\%, en comparación con un aumento del 16,3\% en el índice re-construido con cambio y del $15,1 \%$ en el ICV sin cambio: todos son valores relativamente similares que ayudan a corroborar la afirmación de que la divergencia de 1919 en el gráfico 4 se relaciona con la falta de empalme de la serie oficial. ¿Por qué no se empalmó la serie oficial? Dado el saber numérico de Bunge comentado en la sección anterior, es difícil creer que dicha falta de empalme sea por falta de conocimiento estadístico. En los artículos de 1919 y 1920 de la REA, la alteración en las participaciones se produjo en 1918, justificada por el aumento del 30\% en el alquiler. Por ello, en 1919 el ICV aumentó (Valle y Ferrari, 1920: 261). El informe de la DGEN de 1924 y el artículo de la REA de 1928 (Bunge, 1928) colocaron el cambio en 1919, lo cual genera una disminución interanual en 1919. 1919 fue el año de las revueltas sociales de la Semana Trágica, que, junto con episodios similares posteriores, ${ }^{29}$ son un signo de la debilidad del presidente Yrigoyen. Dadas sus repercusiones ${ }^{30}$ y los conflictos laborales que se sucedieron, ${ }^{31}$ la falta de empalme y el año elegido para la alteración de la participación de los capítulos podrían asociarse con estos eventos políticos. En enero de 1919, el periódico socialista La Vanguardia acusaba al gobierno radical de maniobras electorales en la Ciudad de Buenos Aires relacionadas con la modificación del precio del pan. ${ }^{32}$ En agosto de dicho año, el mismo diario sostenía que "el pan a 40 centavos (...) cuando los demás artículos de consumo están por las nubes (...) es un escándalo intolerable, un verdadero atentado contra la salud de la clase popular." ${ }^{33}$ En junio de 1920, La Vanguardia denuncia una "farsa que el gobierno está representando con sus pretendidos "desvelos" por combatir la carestía de la vida."34 En 1919 y 1924 los presidentes eran diferentes, pero pertenecían al mismo partido político,

29 Entre las más relevantes se encuentran las huelgas en la provincia de Santa Cruz (1920-1922), conocidas como la Patagonia rebelde (Bayer, 1974).

30 Por ejemplo, la Liga Patriótica, una organización paramilitar que agrupó sectores reaccionarios de la sociedad argentina, surgió después de los acontecimientos de la Semana Trágica (Rock, 1997).

31 A principios de 1924, por ejemplo, los principales sindicatos convocaron a una huelga general contra la Ley de Pensiones propuesta por el Ejecutivo (Anapios, 2013).

32 (28 de enero de 1919), ¿ Nuevamente el pan bazo?, La Vanguardia, Buenos Aires, p. 1.

33 (4 de agosto de 1919), El Pan, La Vanguardia, Buenos Aires, p. 1.

34 (24 de junio de 1920), Impuestos y carestía de la vida, La Vanguardia, Buenos Aires, p. 1. 
la Unión Cívica Radical. Por ello, incrementos menos sustanciales en el costo de vida en períodos de conflicto social le brindarían un significado diferente a dichos eventos. Teniendo en cuenta únicamente la evolución del ICV, el prolongado conflicto y su extensión a lo largo del país sugieren, por ejemplo, un descontento general con las administraciones radicales, ya que los precios disminuyeron bruscamente entre 1920 y 1923 en la Ciudad de Buenos Aires, como se muestra en el gráfico 4. También puede ser considerado una prueba más de la disparidad en la evolución de los precios a lo largo de todo el país, lo que no se refleja en un ICV basado en las fluctuaciones de los precios en la Ciudad de Buenos Aires. Estas divergencias regionales se presentaron anteriormente, donde se demostró que, a pesar de lo que Bunge afirmó, los precios variaron de forma diferente en todo el país. Cuesta (2012; 2016a) discute las diferencias regionales de precios, mientras que Harriague y Rayes (2018) destacan la carencia de índices nacionales. Con este trabajo se continúa en la línea de resaltar el alcance geográfico del ICV de Bunge, considerado y utilizado por la gran parte de la literatura como un índice nacional, y la posibilidad de diferencias regionales.

\section{Reflexiones finales}

Las estadísticas suelen percibirse como hechos incontestables y apolíticos, que viajan en el tiempo, y que reflejan y establecen la realidad. Platt argumenta que los individuos deberían cuestionarlas, mientras que la sociología de la cuantificación demuestra que las estadísticas no son objetivas ni neutrales. Las estadísticas - en tanto conocimiento económico - son construcciones dinámicas que se adaptan al contexto. Al estudiar cómo y por qué se producen y utilizan, pueden concebirse como science in the making. Un procedimiento útil para estudiarlas es la metodología de de-construcción/construcción/re-construcción de estadísticas. Este trabajo aplica dicha metodología al primer ICV argentino, elaborado por Alejandro Bunge en las primeras décadas del siglo XX. Como tal, es el primer análisis exhaustivo del ICV de Bunge, un indicador que hasta el momento ha viajado en el tiempo sin ser cuestionado. Asimismo, demuestra que la estimación tenía varios problemas, que sólo son evidentes si la misma se analiza siguiendo la metodología de tres etapas.

El análisis de los problemas de dicho ICV demuestra que Bunge no tomó decisiones metodológicas por falta de conocimiento, lo cual indica cómo el criterio, el saber, y las opiniones individuales afectan las estadísticas. A veces sus decisiones eran deliberadas: al explicar los números índice, Bunge se centró en métodos que le permitieron estimar el "costo de un grupo de artículos, prescindiendo de las respectivas cantidades" (1918, p. 42), lo cual es evidencia de que estaba al tanto de la necesidad de tener cantidades para estimar el ICV. El ancla en precios en lugar de en cantidades, por lo tanto, se explica por el objetivo mismo del ICV: ser la base para el coeficiente de corrección monetaria. Como con el uso de las importaciones como indicador del capítulo otros gastos, otras veces Bunge hizo elecciones debido a la disponibilidad de datos, y en base a su comprensión de la 
dinámica económica argentina. En algunos casos, las decisiones se adoptaron utilizando supuestos erróneos, como el uso de precios al por mayor en lugar de precios minoristas. En otros, su opacidad con respecto a las fuentes o a los procedimientos dificulta la comprensión de su razonamiento. Lo que queda claro es que Bunge trató de adaptar las normas internacionales a los datos existentes y / o a la situación argentina y sus limitaciones. En este sentido, contribuyó al desarrollo de números índices en situaciones particulares.

El gráfico 4 muestra que la inexistencia de un empalme apropiado explica gran parte de la divergencia entre el ICV de Bunge y los índices re-construidos. Las discrepancias entre las series re-construidas se relacionan con: los tipos de precios (minoristas versus mayoristas, y un alquiler para la Ciudad de Buenos Aires en lugar de para todo el país si los otros datos utilizados responden a la misma localidad), el impacto diferencial de las participaciones de los capítulos y la metodología utilizada para empalmar series. Las series re-construidas son un ejemplo claro de cómo suposiciones diferentes impactan en los resultados. Siguiendo fielmente los procedimientos de Bunge, tal como muestra el ICV re-construido con cambios, se obtiene una serie que diverge aún más de la oficial. El índice de Bunge re-construido sin cambios es preferible, ya que las encuestas de gastos del DNT de los años veinte muestran que los gastos de alquiler no aumentaron como Bunge asumió. Fue mérito de Bunge haber elaborado un ICV en una época en la cual muchas de las discusiones estadísticas distaban de tener la evidencia y transcendencia que poseen actualmente. ${ }^{35}$ Por ello, cualquier reconstrucción de un índice basada en las fuentes de datos disponibles en la época implica cierto anacronismo ya que los conocimientos estadísticos de los que disponemos hoy son superiores.

¿Cuáles son las posibles implicancias para la historiografía de estas nuevas estimaciones? El impacto de la Primera Guerra Mundial sobre los precios locales fue interpretado en base al ICV de Bunge. La re-construcción muestra que dicho índice aumentó en 1919 en lugar de disminuir, generando importantes diferencias de nivel entre el índice oficial y las re-construcciones. Este diferente nivel impacta en otros índices y series relacionados, como los salarios reales. Por ello, observaciones acerca de los mismos como las de Halperin Donghi (2000), Gerchunoff y Aguirre (2006) y Vence Conti y Cuesta (2014) deben ser discutidas a la luz de las nuevas series. Un mayor ICV implica menores salarios reales y por ende trabajadores con un menor poder adquisitivo, o una mayor carestía de vida, utilizando el vocabulario de la época.

35 Para un análisis de la evidencia y trascendencia del reciente debate en torno al índice de precios argentino ver Daniel y Lanata Briones (2019). 


\section{Q Bibliografía}

" Alonso, W. y P. Starr eds. (1987). The Politics of Numbers. New York: Russell Sage Foundation.

"Amaral, S. (1995). Alta inflación y precios relativos. El pago de las obligaciones en Buenos Aires 1836-1854. Trimestre Económico, 56 (221), pp. 163-221.

"Anapios, L. (2013). La ley de jubilaciones de 1924 y la posición del anarquismo en la Argentina. Revista de Historia del Derecho, 46, pp. 27-43.

" Anderson, M. (1988). The American Census: A Social History. New Haven: Yale University Press.

" Asiain, A. (2014). Alejandro Bunge (1880-1943). Un conservador defensor de la independencia económica y la soberanía nacional. Revista Ciclos, 23 (22), pp. 83-102.

" Bayer, O. (1974). Los vengadores de la Patagonia Trágica. Buenos Aires: Editorial Galerna.

" Bunge, A.E. (1918). Costo de la vida en la Argentina, de 1910 a 1917. Revista de Economía Argentina, 1 (1), pp. 39-63.

" Bunge, A.E. (1919). Costo de la vida en la Argentina. Sus variaciones de 1910 a 1918. Revista de Economía Argentina, 3 (16), pp. 309-32.

"Bunge, A.E. (1920a). The Coefficient of Money Correction: The Use of Index Numbers in the Determination of Fluctuations in the Purchasing Power of Money. Washington D.C.: s./ed.

" Bunge, A.E. (1920b). Los problemas económicos del presente. Buenos Aires: s./ed.

"Bunge, A.E. (1928). El costo de la vida y los salarios en la Argentina (I). Revista de Economía Argentina, 21 (123), pp. 199-207.

" Bunge, A.E. (1940). Una nueva Argentina. Buenos Aires: Hyspamérica.

" Camargo, A.P.R. (2007). Números para o progresso: um panorama da atividade estatística a Primeira Republica. Bulhões de Carvalho, um medico cuidando da estatística brasileira, Rio de Janeiro, 11.

" Crovetto, N. y N. Zeolla (2019). "La crítica a la teoría clásica de las ventajas comparativas y los orígenes del pensamiento propio. Un análisis de las raíces del estructuralismo latinoamericano. Revista Ciclos, 50.

"Cuesta, E. M. (2012). De índices y fuentes. Una revisión sobre la Historia de Precios y Salarios en Buenos Aires. Investigaciones y Ensayos, 61.

"Cuesta, E. M. (2016a). Un acercamiento a la evolución de los precios y salarios en Mendoza y Buenos Aires en el siglo XX. Revista de Historia Económica e Historia de Empresas, 19 (2), pp. 403-437.

"Cuesta, E. M. (2016b). El costo de nivel de vida en la Capital Federal de 1963 y los cambios de paradigmas estadísticos en Argentina. Estadística y Sociedad, 4, pp. 93-108.

" Curtis, B. (2001). The Politics of Population: State Formation, Statistics, and the Census of Canada, 1840-1875. Toronto: University of Toronto Press.

" Damill, M. y R. Frenkel (1990). Hiperinflación y estabilización: la experiencia argentina reciente. Buenos Aires: CEDES.

"Daniel, C. (2009). Un imaginario estadístico para la Argentina moderna. Cuadernos del IDES, 17. 
" Daniel, C. y M. Heredia (2014). Génesis, apogeo y destrucción de un número público: el caso del índice de precios al consumidor en Argentina. Ponencia presentada en las IX Jornadas de Estudios Sociales de la Economía. La dimensión social de los procesos y objetos económicos, Buenos Aires, IDAES.

" Daniel, C. y C.T. Lanata Briones (2019). Battles over numbers: the case of the Argentine consumer price index (2007-2015). Economy and Society, 48(1), pp. 127-151. DOI: 10.1080/03085147.2019.1579438.

" della Paolera, G. (1988). How the Argentine Economy Performed during the International Gold Standard. A Reexamination. Ph.D Dissertation, University of Chicago.

" della Paolera, G. y J. Ortíz (1995). Dinero, intermediación financiera y nivel de actividad en 110 años de historia económica argentina. Documentos de Trabajo, Universidad Torcuato Di Tella.

" Desrosières, A. (1993) 1998. The Politics of Large Numbers: A History of Statistical Reasoning. Cambridge: Harvard University Press.

"Desrosières, A. (2001). How Real Are Statistics? Four Possible Attitudes. Social Research, 68 (2), pp. 339-355.

" Díaz Alejandro, C.F. (1981). Tipos de cambio y términos de intercambio en la República Argentina. 1913-1976. Documentos de Trabajo CEMA, 22.

" Dirección General de Estadísticas de la Nación, (1918). Intercambio económico de la República. 1910-1917, Buenos Aires.

"Dirección General de Estadísticas de la Nación, (1924). El costo de la vida y el poder de compra de la moneda, Buenos Aires.

" Dirección Nacional de Estadísticas y Censos, (1963). Costo del nivel de vida en la Capital Federal. Encuesta sobre condiciones de vida de familias obreras realizada en el año 1960, Buenos Aires.

" Dirección Nacional de Investigaciones, Estadística y Censos (1948). Anuario estadístico de la República Argentina. Comercio exterior, 1947, Buenos Aires.

" Di Tella (1964). Precios unitarios de artículos de consumo y servicios, Capital Federal y provincias. 1901-1963. Primera parte, Buenos Aires: Di Tella.

" Fernández López, M. (1994). La estabilidad monetaria: Fisher, Bunge y Prebisch. Anales de la Asociación Argentina de Economía Política, 3 (29), pp. 665-75.

" Fourcade, M. (2009). Economists and Societies. Discipline and Profession in the United States, Britain, and France, 1890s to1990s. Princeton: Princeton University Press.

"Francis, J. A. (2013). The Terms of Trade and the Rise of Argentina in the Long Nineteenth Century. Ph. D. Thesis, London School of Economics and Political Science.

" Furner, M.O. y B. Supple. (1990). Ideas, Institutions, and State in the United States and Britain: An Introduction. En The State and Economic Knowledge. The American and British Experiences, 3-39. Cambridge: Woodrow Wilson Center Press.

" Galiani, S. y P. Gerchunoff (2003). The Labour Market. En A New Economic History of Argentina, pp. 122-69. Cambridge: Cambridge University Press.

" Gerchunoff, P. y H. Aguirre (2006). La economía argentina entre la gran guerra y la gran depresión, Serie Estudios y Perspectivas, 32.

" González Bollo, H. (2012). La teodicea estadística de Alejandro E. Bunge (1880-1943), Buenos Aires:Imago Mundi.

" González Bollo, H. (2014). La fábrica de las cifras oficiales del Estado argentino (1869- 
1947). Buenos Aires:Universidad de Quilmes.

" Hacking, I. (1990) 2010. The Taming of Chance. Cambridge:Cambridge University Press.

" Halperin Donghi, T. (2000). Vida y Muerte de la República Verdadera (1910-1930). Buenos Aires: Ariel.

" Harper, R. H. R. (1998). Inside the IMF. An Ethnography of Documents, Technology and Organisational Action, San Diego:Academic Press.

" Harriague, M. M. y A. Rayes (2018). Fuentes para el estudio de la historia económica argentina. En Nueva historia económica de la Argentina, pp. 243-275. Buenos Aires: Edhasa.

" Hayes, M. (2011). The Social History of Quantifying Inflation: A Sociological Critique. Journal of Economic Issues, 65(1), pp. 97-111.

" IEERAL (1986). Estadísticas de la evolución económica de la Argentina, 1913-1984. Estudios, 9 (39).

" Instituto de Estadística (1939). Precio medio de la habitación ofrecida en la ciudad de Buenos Aires. Revista de Economía y Estadística, 1 (4), pp. 483-514.

" Inter-American Statistical Institute (IASI) (1941). Statistical Activities of the American Nations. 1940. Washington DC.

" Irigoin, M.A., (2000). Inconvertible Paper Currency: Inflation and Economic Performance in Early 19th Century Argentina. Journal of Latin American Studies, 33 (2), pp. 333-59.

"Jany-Catrice, F. (2018). Conflicts in the Calculation and Use of the Price Index: The Case of France. Cambridge Journal of Economics, 42 (4), pp. 963-86.

" Kuntz-Ficker, S. y A. Rayes (2017). The Contribution of Argentine Exports to the Economy, 1875-1929. En The First Export Era Revisited. Reassessing its contribution to Latin American economies, pp. 39-74. London: Palgrave.

" Lanata Briones, C. T. (2016). Constructing Public Statistics: The History of the Argentine Cost of Living Index, 1918-1943. Ph. D. Thesis, London School of Economics and Political Science. http://etheses.Ise.ac.uk/3319/

" Lanata Briones, C. T. (en prensa a). Constructing Cost of Living Indices: Ideas and Individuals, Argentina, 1918-1935. History of Political Economy.

" Lanata Briones, C. T. (en prensa b). De-construyendo, construyendo y re-construyendo estadísticas: el índice del costo de la vida, Argentina 1933-1937. Anuario IEHS.

"Latour, B. (1987) 2003. Science in Action: How to Follow Scientists and Engineers Through Society. Cambridge: Harvard University Press.

" Llach, J. J. (1985). La Argentina que no fue, Buenos Aires: IDES.

" Loveman, M. (2014). National Colors: Racial Classification and the State in Latin America. New York:Oxford University Press.

" Lucchini, M.C. et al (2000). El pensamiento industrialista argentino en el período de entreguerras-el estudio de un caso: la influencia de List en Bunge. Estudios Interdisciplinarios de América Latina y el Caribe, 11(2).

"Neiburg, F. (2006). Inflation: Economists and Economic Cultures in Brazil and Argentina. Comparative Studies in Society and History, 48 (3), pp. 604-33.

" O’Neill, R., J. Ralph y P.A. Smith (2017). Inflation. History and Measurement. Palgrave Macmillan.

" Otero, H. (2006). Estadística y Nación. Una historia conceptual del pensamiento censal de 
la Argentina moderna. Buenos Aires: Prometeo Libros.

" Pantaleón, J. (2004). El surgimiento de la nueva economía argentina: el caso Bunge. In Intelectuales y expertos. La constitución del conocimiento social en la Argentina, 175-201. Buenos Aires: Ediciones Paidós.

" Pantaleón, J. (2009). Una nación a medida. Creencia económica y estadística en la Argentina (1918-1952), Buenos Aires: Ediciones Al Margen.

" Patriarca, S. (1996). Numbers and Nationhood: Writing Statistics in Nineteenth-Century Italy. Cambridge:Cambridge University Press.

" Platt, D.C.M. (1989). Mickey Mouse Numbers in World History: The Short View. Basingstoke: Palgrave Macmillan.

" Porter, T.M. (1995). Trust in Numbers: The Pursuit of Objectivity in Science and Public Life. Princeton: Princeton University Press.

"Prévost, J.G. y J.P. Beaud (2012). Statistics, Public Debate and the State, 1800-1945: A Social, Political and Intellectual History of Numbers. London: Routledge.

" Randall, L. (1976). Lies, Damn Lies, and Argentine GDP. Latin American Research Review, 11 (1), pp. 137-58.

" República Argentina. Departamento Nacional de Trabajo (1915). Boletín del Departamento Nacional de Trabajo. Anuario estadístico del trabajo. Año 1913, 30.

" República Argentina. Departamento Nacional de Trabajo (1916). Boletín del Departamento Nacional de Trabajo. Anuario estadístico del trabajo. Año 1914, 33.

" República Argentina. Departamento Nacional de Trabajo (1918). Boletín del Departamento Nacional de Trabajo. Precios de los artículos de primera necesidad, 38, pp. 179-86.

" República Argentina. Departamento Nacional de Trabajo (1920). Crónica Mensual del Departamento Nacional del Trabajo (1920). Presupuestos obreros, 3 (32), pp. 507-8.

" República Argentina. Departamento Nacional de Trabajo (1922). Crónica Mensual del Departamento Nacional de Trabajo (1922). Productos alimenticios, 5 (52), pp. 833-44.

" República Argentina. Departamento Nacional de Trabajo (1926). Crónica Mensual del Departamento Nacional de Trabajo (1926). Recursos y gastos de la familia obrera; año 1925, 9 (97), p. 1735.

" República Argentina. Departamento Nacional de Trabajo (1927). Crónica Mensual del Departamento Nacional de Trabajo (1927). Los presupuestos obreros y su evolución en la Capital Federal en el quinquenio 1922-1926, 10 (109), pp. 1958-9.

" República Argentina. Departamento Nacional de Trabajo (1929). Crónica Mensual del Departamento Nacional de Trabajo (1929). Recursos, gastos y vivienda de la familia obrera de la Capital Federal, en el año 1928, 12 (133), pp. 2695-700.

" República Argentina. Departamento Nacional de Trabajo (1930). Crónica Mensual del Departamento Nacional de Trabajo (1930). Recursos, gastos y vivienda de la familia obrera de la Capital Federal, en el año 1929, 13 (147), pp. 3142-6.

" República Argentina.Departamento Nacional de Trabajo (1935). Costo de la Vida. Presupuestos familiares. Precios de artículos de primera necesidad. Índices del costo de la vida.

" República Argentina. Departamento Nacional del Trabajo (1937). Condiciones de vida de la familia obrera. Investigaciones Especiales. Serie C, 8.

"Investigaciones Especiales. Serie C, 1. 
" Revista de Economía Argentina (1921). Artículos de primera necesidad, 6 (31), pp. 60-1.

" Rock, D., (1997). El radicalismo argentino, 1890-1930. Buenos Aires: Amorrotu.

"Salvatore, R.D. (2010). Better-off in the Thirties: Welfare Indices for Argentina, 1900-1940. En Living Standards in Latin American History, pp. 127-66. Cambridge: Harvard University Press.

" Senra, N. (2011). As instituições estatísticas como centros de ciência, uma (r)evolução necessária. Estatística e Sociedade, 1, pp. 49-64.

"Schwartzman, S. (1997). Legitimidade, controvérsias e traduções em estatísticas públicas. Teoria \& Sociedade, 2, pp. 9-38.

"Scott, J.W. (1988). A Statistical Representation of Work. La Statistique de I'Industrie à Paris, 1847-1848. En Gender and the Politics of History, 113-98. New York:Columbia University Press.

" Searle, R., (2015). Is There Anything Real about Real Wages? A History of the Official British Cost of Living Index, 1914-62. Economic History Review, 68 (1), pp. 145-66.

"Sember, F. (2013). The Reception of Irving Fisher in Argentina: Alejandro Bunge and Raúl Prebisch. The European Journal of History of Economic Thought, 20 (2), pp. 372-98.

"Stapleford, T.A. (2009). The Cost of Living in America: A Political History of Economic Statistics, 1880-2000. New York: Cambridge University Press.

"Sturzenegger, A. y R. Moya (2003). Economic Cycles. En A New Economic History of Argentina, pp. 87-113. Cambridge: Cambridge University Press.

" Tena-Junguito, A. y H. Willebald (2013). On the Accuracy of Export Growth in Argentina, 1870-1913. Economic History of Developing Regions, 28(1), pp. 28-68.

" Touchelay, B. (2015). La fabuleuse histoire de l'indice des prix de détail en France. Entreprises et Histoire, 2(79), pp. 135-146.

" Tooze, A.J. (2001). Statistics and the German State, 1900-1945: The Making of Modern Economic Knowledge. Cambridge:Cambridge University Press.

" Tooze, A.J. (2008). Trouble with Numbers: Statistics, Politics, and History in the Construction of Weimar's Trade Balance, 1918-1924. The American Historical Review, 113 (3), pp. 678-700.

"Valle, J.C. y L.A. Ferrari (1920). Costo de la vida en la Argentina de 1910 a 1919. Revista de Economia Argentina, 4 (22), pp. 256-61.

"Vence Conti, A. y E.M. Cuesta (2014). Políticas laborales y salarios durante el primer radicalismo y el primer peronismo (1916-1955). Revista de Economía Política e Historia Económica, 10 (32), pp. 275-300.

"Villanueva, J. (1964). The Inflationary Process in Argentina, 1943-1962. Buenos Aires, s/ ed.

"Vitelli, G. (1986). Cuarenta años de inflación en la Argentina. Buenos Aires: Hyspamérica.

"Williamson, J.G. y A.M. Taylor (1994). Convergence in the Age of Mass Migration. NBER Working Paper, 4711. 


\section{Q Apéndice}

CUADRO 5. Estimaciones re-construidas del ICV de Bunge, 1912-1932.

Base: 1912=100. Fuente: elaboración propia.

\begin{tabular}{|r|rr|}
\hline & $\begin{array}{r}\text { ICV re-construido } \\
\text { con cambio }\end{array}$ & $\begin{array}{r}\text { ICV re-construido } \\
\text { sin cambio }\end{array}$ \\
\hline $\mathbf{1 9 1 2}$ & 100.0 & 100.0 \\
$\mathbf{1 9 1 3}$ & 103.5 & 103.5 \\
$\mathbf{1 9 1 4}$ & 109.7 & 109.7 \\
$\mathbf{1 9 1 5}$ & 118.9 & 118.9 \\
$\mathbf{1 9 1 6}$ & 127.3 & 127.3 \\
$\mathbf{1 9 1 7}$ & 142.3 & 142.3 \\
$\mathbf{1 9 1 8}$ & 175.3 & 175.3 \\
$\mathbf{1 9 1 9}$ & 192.2 & 189.2 \\
$\mathbf{1 9 2 0}$ & 223.7 & 217.9 \\
$\mathbf{1 9 2 1}$ & 204.2 & 196.7 \\
$\mathbf{1 9 2 2}$ & 180.3 & 171.1 \\
$\mathbf{1 9 2 3}$ & 171.4 & 162.1 \\
$\mathbf{1 9 2 4}$ & 186.8 & 177.0 \\
$\mathbf{1 9 2 5}$ & 181.8 & 171.9 \\
$\mathbf{1 9 2 6}$ & 178.0 & 168.2 \\
$\mathbf{1 9 2 7}$ & 176.6 & 166.9 \\
$\mathbf{1 9 2 8}$ & 174.0 & 164.4 \\
$\mathbf{1 9 2 9}$ & 176.6 & 166.9 \\
$\mathbf{1 9 3 0}$ & 179.3 & 169.5 \\
$\mathbf{1 9 3 1}$ & 153.7 & 145.3 \\
$\mathbf{1 9 3 2}$ & 136.2 & 128.7 \\
\hline
\end{tabular}

